\title{
Coprophilous pyrenomycetes s.l. from the Tuscan Archipelago and adjacent peninsular coast: description of five species new to Italy
}

\author{
Doveri $\mathbf{F}^{1}$ \\ ${ }^{I}$ via dei Funaioli 22, I 57126 Livorno, Italy, f.doveri@sysnet.it
}

Doveri F 2014 - Coprophilous pyrenomycetes s.l. from the Tuscan Archipelago and adjacent peninsular coast: description of five species new to Italy. Mycosphere 5(1), 188-216, Doi 10.5943/mycosphere/5/1/10

\begin{abstract}
All author's collections of 55 pyrenomycetes s.l. so far known from dung in Tuscan coast and islands (Italy) are listed. Lophotrichus macrosporus, Pleospora ambigua, Rhytidospora cainii, Sporormiella isomera, Westerdykella cylindrica are described and discussed. A world-key to Rhytidospora species is provided.
\end{abstract}

Key words - dichotomous key - dung - geographical coordinates - records

\section{Introduction}

This article is a continuation of my previous studies on rare or new coprophilous discomycetes from the Tuscan archipelago (Doveri 2012).

The area under study is bounded by a line joining the following three geographical coordinates, $41^{\circ} 45^{\prime} 56^{\prime \prime} \mathrm{N} 9^{\circ} 43^{\prime} 05^{\prime \prime} \mathrm{E}$; $42^{\circ} 22^{\prime} 51^{\prime \prime} \mathrm{N} 11^{\circ} 25^{\prime} 50^{\prime \prime} \mathrm{E}$; $44^{\circ} 02^{\prime} 34^{\prime \prime} \mathrm{N} 10^{\circ} 01^{\prime} 10^{\prime \prime} \mathrm{E}$, and includes the islands of the Tuscan Archipelago, in Northern Tyrrhenian Sea, and the coast of the Tuscan provinces of Massa, Lucca, Pisa, Livorno and Grosseto, facing this sea. Climate, flora and fauna are typically Mediterranean.

The aim of this paper is to update the list of pyrenomycetes from this area, specifying their precise location and dung source, and to describe and widely comment on some pyrenomycetes new to Italy.

\section{Materials \& Methods}

All collections were obtained from different types of dung cultured in non-axenic damp chambers, and studied according to the methods of Richardson \& Watling (1997) and Richardson (2001), slightly modified by Doveri (2004).

Abbreviations: $\mathrm{AMB}=$ Herbarium of Bresadola Mycological Association; CLSM $=$ author's personal herbarium; d.c. $=$ damp chamber culture; MCVE $=$ Herbarium of Venetian Civic Museum.

\section{Results}

This census and study of coprophilous fungi from the Tuscan coast and archipelago fall into

Submitted 27 January 2014, Accepted 11 February 2014, Published online 25 February 2014 
a broader survey I have now studied coprophilous ascomycetes and basidiomycetes from Italy (Cacialli et al. 1995, Doveri 2004, 2011) for more than 25 years. During this period I have identified in the field or isolated in damp chamber 176 pyrenomycetes s.l. from Italy, with 55 detailed below from the Tuscan coast and archipelago:

Arnium arizonense (Griffiths) N. Lundq. \& J.C. Krug

Livorno, Botro delle Fontanelle, 43d 30' 40.7376" 10d 22' 56.2614", 200 m, on cattle dung in d.c., F. Doveri, 5.1995, MCVE 484.

\section{Cercophora mirabilis Fuckel}

Livorno, Botro delle Fontanelle, 43d 30' 40.7376" 10d 22' 56.2614", 200 m, on cattle dung, F. Doveri, 14.12.94, CLSM 02894.

\section{Chaetomium bostrychodes Zopf}

1) Livorno, Quercianella, 43d 27' 49.521" 10d 22' 47.7654", $0 \mathrm{~m}$, on toad dung in d.c., C. Doveri, 10.97, 284.3-Rosignano Marittimo, CLSM 02197 penta. 2) Grosseto, Principina terra, 42d 43' 14.8146" 11d 1' 42.189", 0 m, on rabbit dung in d.c., F. Doveri, CLSM 02197 epta. 3) Grosseto, Principina terra, 42d 43' 14.8146" 11d 1' 42.189", 0 m, on wild pig dung in d.c., F. Doveri, CLSM 02197 octo. 4) Grosseto, Punta Ala, 42d 48' 21.063" 10d 44' 38.1942", 0 m, on hedgehog dung in d.c., F. Doveri and F. Bersan, 2.4.98, CLSM 02197 ena. 5) Livorno, 43d 31' 45.5484" 10d 21' 7.9626", 0 m, on snail dung in d.c., F. Doveri, 20.9.98, CLSM 02197-XIII. 6) Pisa, S. Rossore Park, 43d 43' 18.2418" 10d 17' 55.1502", 0 m, on wild rabbit dung in d.c., F. Doveri, 5.3.00, CLSM 02197-XXVIII. 7) Livorno, Corbolone, 43d 33' 19.6272" 10d 22' 31.0764", $150 \mathrm{~m}$, on horse dung in d.c., G. Cacialli and F. Doveri, 14.4.00, CLSM 02197-XXIX. 8) Grosseto, Follonica, 42d 55' 59.6568" 10d 43' 41.6454", 0 m, on fallow-deer dung in d.c., F. Doveri, 15.5.05, CLSM 02197-LIX. 9) Livorno, Palazzi di Cecina, 43d 19' 40.7532" 10d 29' 53.2422", 0 m, on cattle dung in d.c., F. Doveri, 30.4.06, CLSM 02197-LXI. 10) Livorno, 43d 31' 45.5484" 10d 21' 7.9626", $30 \mathrm{~m}$, on gecko dung in d.c., M. Seu, 2.9.08, CLSM 02197-LXVII. 11) Livorno, Montioni, 43d 1' 57.7086" 10d 45' 1.674", 50 m, on sheep dung in d.c., F. Doveri, 18.3.09, CLSM 02197-LXX. 12) Livorno, Montioni, 43d 1' 57.7086" 10d 45' 1.674", 50 m, on rabbit dung in d.c., F. Doveri, 18.3.09, CLSM 02197-LXXI. 13) Livorno, Elba island, Campo nell'Elba, Pradazzo-Valle Buia, 42d 44' 41.64" 10d 10' 15.3732", 200 m, on wild pig dung in d.c., C. Cotta, 5.10.10, CLSM 02197-LXXII. 14) Livorno, Elba island, Campo nell'Elba, Pradazzo-Valle Buia, 42d 44' 41.64" 10d 10' 15.3732", 200 m, on marten dung in d.c., C. Cotta, 5.7.13, CLSM 02197-LXXIII. 15) Livorno, Quercianella, 43d 27' 49.521" 10d 22' 47.7654", $50 \mathrm{~m}$, on roe deer dung in d.c., F. Doveri, 23.12.12, CLSM 02197LXXV. 16) Livorno, Quercianella, 43d 27' 49.521" 10d 22' 47.7654", $0 \mathrm{~m}$, on wild pig dung in d.c., F. Doveri, 31.5.13, CLSM 02197-LXXVII. 17) Livorno, Elba island, Campo nell'Elba, PradazzoValle Buia, 42d 44' 41.64" 10d 10' 15.3732", 200 m, on hare dung in d.c., A. Pierulivo, 10.8.13, CLSM 02197-LXXX.

Total 17: wild pig 3; rabbit 2; cattle 1; fallow deer 1; gecko 1; hare 1; hedgehog 1; horse 1; marten 1; roe deer 1; sheep 1; snail 1; toad 1; wild rabbit 1

Chaetomium brasiliense Bat. \& Pontual

Livorno, Quercianella, 43d 27' 49.521" 10d 22' 47.7654", 0 m, on fallow deer dung in d.c., F. Doveri, 22.12.12, CLSM 001.13.

\section{Chaetomium funicola Cooke}

Livorno, Suvereto, 43d 3' 50.3856" 10d 39' 31.6728", 0 m, on horse dung in d.c., F. Doveri, 18.3.09, CLSM 021.02 ter. 
Chaetomium globosum Kunze : Fr

1) Livorno, Elba island, $m$ ?, on wild rabbit dung in d.c., L. Levorato, 9.4.11, CLSM 001.03-XVII. 2) Livorno, Capraia island, 43d 2' 6" 9d 50' 36", 0 m, on hedgehog dung in d.c., L. Levorato, 11.4.11, CLSM 001.03-XVIII. 3) Livorno, Elba island, Campo nell'Elba, Pradazzo-Valle Buia, 42d 44' 41.64" 10d 10' 15.3732", 200 m, on marten dung in d.c., C. Cotta, 5.7.13, CLSM 001.03-XIX.

Total 3: hedgehog 1; marten 1; wild rabbit 1

\section{Chaetomium murorum Corda}

1) Livorno, Montioni, 43d 1' 57.7086" 10d 45' 1.674" 0 m, on rabbit dung in d.c., F. Doveri, 18.3.09, CLSM 010.08 quater. 2) Livorno, Elba island, m ?, on sheep dung in d.c., L. Levorato, 9.4.11, CLSM 010.08-XXIV. 3) Livorno, Capraia island, 43d 2' 6" 9d 50' 36", 0 m, on hedgehog dung in d.c., L. Levorato, 11.4.11, CLSM 010.08-XXV.

Total 3: hedgehog 1; rabbit 1; sheep 1

\section{Chaetomium robustum L.M. Ames}

1) Livorno, Pianosa island, 42d 35' 3.501" 10d 4' 46.6068", 0 m, on wild rabbit dung in d.c., L. Levorato, 8.4.11, CLSM 008.02-XIV. 2) Grosseto, Giglio island, Castello, 42d 21' 58.608" 10d 54' 1.7886", 400 m, on wild rabbit dung in d.c., S. Rum, 22.3.12, CLSM 008.02-XV. 3) Livorno, Elba island, ?, on sheep dung in d.c., L. Levorato, 9.4.11, CLSM 008.02-XVI. 4) Livorno, Elba island, ?, on wild rabbit dung in d.c., L. Levorato, 9.4.11, CLSM 008.02-XVII. 5) Livorno, Capraia island, 43d 2' 6" 9d 50' 36", 0 m, on sheep dung in d.c., L. Levorato, 11.4.11, CLSM 008.02-XVIII. 6) Livorno, Quercianella, 43d 27' 49.521" 10d 22' 47.7654", $0 \mathrm{~m}$, on fallow deer dung in d.c., F. Doveri, 22.12.12, CLSM 008.02-XX. 7) Livorno, Capraia island, 43d 2' 6" 9d 50' 36" 0 m, on hedgehog dung in d.c., L. Levorato, 11.4.11, CLSM 008.02-XXI. 8) Livorno, Elba island, Campo nell'Elba, Pradazzo-Valle Buia, 42d 44' 41.64" 10d 10' 15.3732", 200 m, on mouflon dung in d.c., C. Cotta, 5.10.10, CLSM 008.02-XXII. 9) Livorno, Quercianella, 43d 27' 49.521" 10d 22' 47.7654", $0 \mathrm{~m}$, on wild pig dung in d.c., F. Doveri, 31.5.13, CLSM 008.02-XXIII.

Total 9: wild rabbit 3; sheep 2; fallow deer 1; hedgehog 1; mouflon 1; wild pig 1

\section{Chaetomium semen citrulli Sergeeva}

Livorno, Elba island, Campo nell'Elba, Pradazzo-Valle Buia, 42d 44' 41.64" 10d 10' 15.3732", 200 m, on marten dung in d.c., C. Cotta, 5.7.13, CLSM 05798 quater.

\section{Chaetomium succineum L.M. Ames}

Livorno, Elba island, ? m, on sheep dung in d.c., L. Levorato, 8.4.11, CLSM 003.12.

\section{Delitschia winteri Plowr. ex G. Winter}

1) Grosseto, Maremma Park, 42d 37' 17.2338" 11d 5' 33.9282", 0 m, on fallow deer dung in d.c., E. Bizio and M. Zugna, 16.3.96, MCVE 537. 2) Grosseto, Giglio island, Castello, 42d 21' 58.608" 10d 54' 1.7886", 400 m, on wild rabbit dung, S. Rum, 22.3.12, CLSM 01396-XIII.

Total 2: fallow deer 1; wild rabbit 1

Enterocarpus grenotii Locq.-Lin.

Pisa, Calambrone stables, 43d 36' 20.4876" 10d 18' 48.6036", 0 m, on horse dung in d.c., F. Doveri, 15.4.97, MCVE 579.

\section{Hypocopra brefeldii Zopf}

Livorno, Pianosa island, 42d 35' 3.501" 10d 4' 46.6068", 0 m, on wild rabbit dung in d.c., L. Levorato, 8.4.11, CLSM 01997 ter. 
Kernia cauquensis Calviello

Livorno, Montioni, 43d 1' 57.7086" 10d 45' 1.674", $50 \mathrm{~m}$ a.s.l., on sheep dung in d.c., F.

Doveri, 18.3.09, CLSM 006.09.

\section{Lophotrichus macrosporus}

1) Livorno, Elba island, ?, ? m, on sheep dung in d.c., L. Levorato, 9.4.11, CLSM 004.12. 2) Livorno, Capraia island, 43d 2' 6" 9d 50' 36", 0 m, on hedgehog dung in d.c., L. Levorato, 11.4.11., CLSM 004.12 bis.

Total 2: hedgehog 1; sheep 1

Pleospora ambigua (Berl. \& Bres.) Wehm. var. ambigua

Lucca, Viareggio, 43d 52' 48.9462" 10d 13' 56.9166", 0 m, on horse dung, G. Cacialli, 30.3.13, CLSM 006.13.

Pleospora herbarum (Pers.: Fr.) Rabenh.

Lucca, Viareggio, 43d 52' 48.9462" 10d 13' 56.9166", 0 m, on horse dung, G. Cacialli, 30.3.13, CLSM 019.01 bis.

Podospora anserina (Ces. ex Rabenh.) Niessl

1) Grosseto, Principina terra, 42d 43' 14.8146" 11d 1' 42.189", $0 \mathrm{~m}$, on rabbit dung in d.c., F. Doveri, 2.4.98, CLSM 02697 bis. 2) Livorno, Palazzi di Cecina, 43d 19' 40.7532" 10d 29' 53.2422", $0 \mathrm{~m}$, on cattle dung in d.c., F. Doveri, 30.4.06, CLSM 02697-XXIV. 3) Livorno, Quercianella, 43d 27' 49.521" 10d 22' 47.7654", 0 m, on fallow deer dung, F. Doveri, 23.11.12, CLSM 02697-XXXI.

Total 3: cattle 1; fallow deer 1; rabbit 1

Podospora communis (Speg.) Niess1

Livorno, Suvereto, 43d 3' 50.3856" 10d 39' 31.6728", 0 m, on horse dung in d.c., F. Doveri, 18.3.09, CLSM 02897-XXVII.

\section{Podospora curvicolla (G. Winter) Niessl}

1) Livorno, Elba island, Campo nell'Elba, Pradazzo-Valle Buia, 42d 44' 41.64" 10d 10' 15.3732", $200 \mathrm{~m}$, on mouflon dung in d.c., C. Cotta, 5.10.10, CLSM 027.00 octa. 2) Grosseto, Giglio island, Castello, 42d 21' 58.608" 10d 54' 1.7886", 400 m, on wild rabbit dung in d.c., S. Rum, 22.3.12, CLSM 027.00-X.

Total 2: Mouflon 1; wild rabbit 1

Podospora decipiens (G. Winter ex Fuckel) Niess1

1) Pisa, S. Rossore Park, 43d 43' 18.2418" 10d 17' 55.1502", $0 \mathrm{~m}$, on fallow deer dung in d.c., F. Doveri, 5.3.00, CLSM 01397-XXVI. 2) Livorno, Palazzi di Cecina, 43d 19' 40.7532" 10d 29' 53.2422", 0 m, on cattle dung in d.c., F. Doveri, 30.4.06, CLSM 01397-LXXXIII. 3) Livorno, Suvereto, 43d 3' 50.3856" 10d 39' 31.6728", 0 m, on horse dung in d.c., F. Doveri, 18.3.09, CLSM 01397-CIV.

Total 3: cattle 1; fallow deer 1; horse 1

Podospora fimiseda (Ces. \& De Not.) Niess1

Livorno, Botro delle Fontanelle, 43d 30' 40.7376" 10d 22' 56.2614", 200 m, on cattle dung in d.c., F. Doveri, 6.95, MCVE 483.

\section{Podospora gigantea Mirza \& Cain}

Livorno, Botro delle Fontanelle, 43d 30' 40.7376" 10d 22' 56.2614", 200 m, on cattle dung, 
F. Doveri, 26.12.95, Herbarium Horti Botanici Pisani PI-HM-A1.

Podospora granulostriata N. Lundq.

Livorno, Quercianella, 43d 27' 49.521" 10d 22' 47.7654", 0 m, on fallow deer dung in d.c., F. Doveri, 23.12.12, CLSM 021.00 esa.

Podospora myriaspora (H. Crouan \& P. Crouan) Niessl

Pisa, Calambrone stables, 43d 36' 20.4876" 10d 18' 48.6036", 0 m, on horse dung in d.c., F. Doveri, 26.2.97, CLSM 00497.

Podospora pleiospora (G. Winter) Niessl

1) Livorno, Elba island, Campo nell'Elba, Pradazzo-Valle Buia, 42d 44' 41.64" 10d 10' 15.3732", $200 \mathrm{~m}$, on mouflon dung in d.c., C. Cotta, 5.10.10, CLSM 05598-XXI. 2) Grosseto, Giglio island, Castello, 42d 21' 58.608" 10d 54' 1.7886", $400 \mathrm{~m}$, on wild rabbit dung in d.c., S. Rum, 22.3.12, CLSM 05598-XXIV. 3) Livorno, Quercianella, 43d 27' 49.521" 10d 22' 47.7654", 0 $\mathrm{m}$, on fallow deer dung in d.c., F. Doveri, 23.11.12, CLSM 05598-XXV. 4) Livorno, Elba island, Campo nell'Elba, Pradazzo-Valle Buia, 42d 44' 41.64" 10d 10' 15.3732", $200 \mathrm{~m}$, on hare dung in d.c., A. Pierulivo, 10.8.13, CLSM 05598-XXVII.

Total 4: hare 1; fallow deer 1; mouflon 1; wild rabbit 1

Podospora pyriformis (A. Bayer) Cain

Livorno, Botro delle Fontanelle, 43d 30' 40.7376" 10d 22' 56.2614", 200 m, on cattle dung in d.c., F. Doveri, 27.6.96, MCVE 583.

Podospora setosa (G. Winter) Niessl

1) Pisa, Calambrone, 43d 36' 7.6206" 10d 18' 20.523", 0 m, on horse dung in d.c., F. Doveri, 5.5.95, MCVE 489. 2) Pisa, Calambrone, 43d 36' 7.6206" 10d 18' 20.523", 0 m, on horse dung, F. Doveri, 28.11.95, CLSM 00795 bis. 3) Grosseto, Follonica, 42d 55' 59.6568" 10d 43' 41.6454", 0 $\mathrm{m}$, on fallow deer dung in d.c., F. Doveri, 8.7.05, CLSM 00795-XLI. 4) Livorno, BibbonaAgriturismo La Pira, 43d 15' 26.388" 10d 35' 33.0174", 50 m, on horse dung in d.c., F. Doveri, 21.5.06, CLSM 00795-XLVI. 5) Livorno, Bibbona-Agriturismo La Pira, 43d 15' 26.388" 10d 35' 33.0174", $50 \mathrm{~m}$, on sheep dung in d.c., F. Doveri, 21.5.06, CLSM 00795-XLVII. 6) Livorno, Montioni, 43d 1' 57.7086" 10d 45' 1.674", 50 m, on rabbit dung in d.c., F. Doveri, 18.3.09, CLSM 00795-LVI. 7) Livorno, Elba island, Campo nell'Elba, Pradazzo-Valle Buia, 42d 44' 41.64" 10d 10' 15.3732", 200 m, on mouflon dung in d.c., F. Doveri, 5.10.10, CLSM 00795-LIX. 8) Livorno, Elba island, Campo nell'Elba, Pradazzo-Valle Buia, 42d 44' 41.64" 10d 10' 15.3732", 200 m, on wild pig dung in d.c., C. Cotta, 5.10.10, CLSM 00795-LX. 9) Livorno, Pianosa island, 42d 35' 3.501" 10d 4' 46.6068", $0 \mathrm{~m}$, on wild rabbit dung in d.c., L. Levorato, 8.4.11, CLSM 00795-LXV. 10) Livorno, Quercianella, 43d 27' 49.521" 10d 22' 47.7654", $0 \mathrm{~m}$, on fallow deer dung in d.c., F. Doveri, 23.11.12, CLSM 00795-LXVII. 11) Livorno, Quercianella, 43d 27' 49.521" 10d 22' 47.7654", 0 m, on fallow deer dung in d.c., F. Doveri, 19.12.12, CLSM 00795-LXVIII. 12) Livorno, Capraia island, 43d 2' 6" 9d 50' 36", 0 m, on hedgehog dung in d.c., L. Levorato, 11.4.11., CLSM 00795LXIX. 13) Livorno, Quercianella, 43d 27' 49.521" 10d 22' 47.7654", 0 m, on wild pig dung in d.c., F. Doveri, 31.5.13, CLSM 00795-LXXI. 14) Livorno, Quercianella, 43d 27' 49.521" 10d 22' 47.7654", 0 m, on wild pig dung in d.c., F. Doveri, 31.5.13, CLSM 00795-LXXII.

Total 14: fallow deer 3; horse 3; wild pig 3; hedgehog 1; mouflon 1; rabbit 1; sheep 1; wild rabbit 1

Preussia funiculata (Preuss) Fuckel

1) Grosseto, Giglio island, Castello, 42d 21' 58.608" 10d 54' 1.7886", 400 m, on wild rabbit 
dung in d.c., S. Rum, 22.3.12, CLSM 00699 penta. 2) Livorno, Quercianella, 43d 27' 49.521" 10d 22' 47.7654", 0 m, on fallow deer dung in d.c., F. Doveri, 19.12.12, CLSM 00699 esa.

Total 2: fallow deer 1; wild rabbit 1

\section{Preussia isomera Cain}

1) Livorno, Suvereto-Azienda agricola La Suveraia, 43d 3' 13.9248" 10d 39' 12.5532", 50 m, on horse dung in d.c., F. Doveri, 18.3.09, CLSM 002.08 bis. 2) Livorno, Montioni, 43d 1' 57.7086" 10d 45' 1.674", 0 m, on horse dung in d.c., F. Doveri, 18.3.09, CLSM 002.08 ter. 3) Lucca, Viareggio, 43d 52' 48.9462" 10d 13' 56.9166", 0 m, on horse dung, G. Cacialli, 30.3.13, CLSM 002.08 octa.

Total 3: horse 3

Preussia typharum (Sacc.) Cain

Livorno, Montioni, 43d 1' 57.7086" 10d 45' 1.674", 50 m, on cattle dung in d.c., F. Doveri, 18.3.09, CLSM 04098 penta.

\section{Rhytidospora cainii Guarro}

1) Livorno, Quercianella, 43d 27' 49.521" 10d 22' 47.7654", 0 m, on wild pig dung in d.c., F. Doveri, 31.5.13, CLSM 007.13. 2) Livorno, Quercianella, 43d 27' 49.521" 10d 22' 47.7654", 0 m, on wild pig dung in d.c., F. Doveri, 31.5.13, CLSM 007.13 bis.

Total 2: wild pig 2

Schizothecium conicum (Fuckel) N. Lundq.

1) Pisa, Calambrone, 43d 36' 7.6206" 10d 18' 20.523", 0 m, on horse dung in d.c., F. Doveri, 26.2.97, CLSM 00895 penta. 2) Grosseto, Principina terra, 42d 43' 14.8146" 11d 1' 42.189", 0 m, on horse dung in d.c., F. Doveri, 2.4.98, CLSM 00895-XI. 3) Grosseto, Principina terra, 42d 43' 14.8146" 11d 1' 42.189", 0 m, on horse dung in d.c., F. Doveri, 2.4.98, CLSM 00895-XII. 4) Livorno, Palazzi di Cecina, 43d 19' 40.7532" 10d 29' 53.2422", 0 m, on cattle dung in d.c., F. Doveri, 30.4.06, CLSM 00895-LXXVII.

Total 4: horse 3; cattle 1

Schizothecium dakotense (Griffiths) N. Lundq.

Livorno, Elba island, Campo nell'Elba, Pradazzo-Valle Buia, 42d 44' 41.64" 10d 10' 15.3732", 200 m, on hare dung in d.c., A. Pierulivo, 10.8.13, CLSM 014.01 ena.

Schizothecium miniglutinans (Mirza \& Cain) N. Lundq.

Livorno, Elba island, Campo nell'Elba, Pradazzo-Valle Buia, 42d 44' 41.64" 10d 10' 15.3732", 200 m, on mouflon dung in d.c., C. Cotta, 5.10.10, CLSM 01100-XV.

Schizothecium tetrasporum (G. Winter) N. Lundq.

1) Pisa, Calambrone, 43d 36' 7.6206" 10d 18' 20.523", $0 \mathrm{~m}$, on horse dung, F. Doveri, 31.1.96, MCVE 496. 2) Pisa, S. Rossore Park, 43d 43' 18.2418" 10d 17' 55.1502", 0 m, on wild rabbit dung in d.c., F. Doveri, 5.3.00, CLSM 01696 epta. 3) Livorno, Elba island, Campo nell'Elba, Pradazzo-Valle Buia, 42d 44' 41.64" 10d 10' 15.3732", 200 m, on mouflon dung in d.c., C. Cotta, 5.10.10, CLSM 01696-XX. 4) Livorno, Elba island, Campo nell'Elba, Pradazzo-Valle Buia, 42d 44' 41.64" 10d 10' 15.3732", $200 \mathrm{~m}$, on hare dung in d.c., C. Cotta, 5.9.11, CLSM 01696-XXII. 5) Grosseto, Giglio island, Castello, 42d 21' 58.608" 10d 54' 1.7886", $400 \mathrm{~m}$, on wild rabbit dung in d.c., S. Rum, 22.3.12, CLSM 01696-XXIV. 6) Livorno, Elba island, Campo nell'Elba, PradazzoValle Buia, 42d 44' 41.64" 10d 10' 15.3732", 200 m, on hare dung in d.c., A. Pierulivo, 10.8.13, CLSM 01696-XXV. 
Total 6: hare 2; wild rabbit 2; horse 1; mouflon 1

Schizothecium vesticola (Berk. \& Broome) N. Lundq.

1) Pisa, Calambrone, 43d 36' 7.6206" 10d 18' 20.523", $0 \mathrm{~m}$, dozens of gregarious, on horse dung, F. Doveri, 29.1.96, MCVE 495. 2) Grosseto, Principina terra, 42d 43' 14.8146" 11d 1' 42.189", 0 m, on horse dung in d.c., F. Doveri, CLSM 01596 quater. 3) Pisa, S. Rossore Park, 43d 43' 18.2418" 10d 17' 55.1502", 0 m, on fallow deer dung, F. Doveri, 5.3.00, CLSM 01596-XVII. 4) Livorno, Corbolone, 43d 33' 19.6272" 10d 22' 31.0764", $150 \mathrm{~m}$, on horse dung in d.c., G. Cacialli and F. Doveri, 14.4.00, CLSM 01596-XVIII. 5) Livorno, Corbolone, 43d 33' 19.6272" 10d 22' 31.0764", 150 m, on sheep dung in d.c., G. Cacialli and F. Doveri, 14.4.00, CLSM 01596-XIX.

Total 5: horse 3; fallow deer 1; sheep 1

Sordaria fimicola (Roberge) Ces. \& De Not.

1) Pisa, Calambrone, 43d 36' 7.6206" 10d 18' 20.523", 0 m, on horse dung in d.c., F. Doveri, 20.5.95, MCVE 486. 2) Pisa, S. Rossore Park, 43d 43' 18.2418" 10d 17' 55.1502", 0 m, on wild pig dung in d.c., V. Caroti, 26.2.97, CLSM 01195 bis. 3) Grosseto, Principina terra, 42d 43' 14.8146" 11d 1' 42.189", 0 m, on horse dung in d.c., F. Doveri, 2.4.98, CLSM 01195 octo. 4) Livorno, Corbolone, 43d 33' 19.6272" 10d 22' 31.0764", 150 m, on horse dung, G. Cacialli and F. Doveri, 14.4.00, CLSM 01195-XXIII. 5) Grosseto, Follonica, 42d 55' 59.6568" 10d 43' 41.6454", 0 m, on fallow deer dung in d.c., F. Doveri, 8.7.05, CLSM 01195 LXXV. 6) Livorno, Palazzi di Cecina, 43d 19' 40.7532" 10d 29' 53.2422", 0 m, on cattle dung in d.c., F. Doveri, 30.4.06, CLSM 01195LXXX. 7) Livorno, Suvereto-Azienda agricola La Suveraia, 43d 3' 13.9248" 10d 39' 12.5532", 50 $\mathrm{m}$, on horse dung in d.c., F. Doveri, 18.3.09, CLSM 01195-CVII. 8) Livorno, Suvereto, 43d 3' 50.3856" 10d 39' 31.6728", 0 m, on horse dung in d.c., F. Doveri, 18.3.09, CLSM 01195-CVIII. 9) Livorno, Elba island, Campo nell'Elba, Pradazzo-Valle Buia, 42d 44' 41.64" 10d 10' 15.3732", 200 $\mathrm{m}$, on mouflon dung in d.c., F. Doveri, 5.10.10, CLSM 01195-CXX. 10) Livorno, Elba island, Campo nell'Elba, Pradazzo-Valle Buia, 42d 44' 41.64" 10d 10' 15.3732", 200 m, on wild pig dung in d.c., C. Cotta, 5.10.10, CLSM 01195-CXXI. 11) Livorno, Elba island, Campo nell'Elba, Pradazzo-Valle Buia, 42d 44' 41.64" 10d 10' 15.3732", 200 m, on hare dung in d.c., C. Cotta, 5.9.11, CLSM 01195-CXXX. 12) Livorno, Elba island, ?, ? m, on sheep dung in d.c., L. Levorato, 9.4.11, CLSM 01195-CXXXII. 13) Livorno, Elba island, ?, ? m, on sheep dung in d.c., L. Levorato, CLSM 01195-CXXXIII. 14) Livorno, Elba island, ?, ? m, on rabbit dung in d.c., L. Levorato, 9.4.11, CLSM 01195-CXXXIV. 15) Livorno, Capraia island, 43d 2' 6" 9d 50' 36", 0 m, on sheep dung in d.c., L. Levorato, 11.4.11., CLSM 01195-CXXXV. 16) Livorno, Elba island, Campo nell'Elba, Pradazzo-Valle Buia, 42d 44' 41.64" 10d 10' 15.3732", 200 m, on hare dung in d.c., A. Pierulivo, 10.8.13, CLSM 01195-CXXXIX. 17) Grosseto, Montecristo island, 42d 20' 50.3298" 10d 19' 2.2404", ?, on goat dung in d.c., J. Viacava, 10.5.13, CLSM 01195-CXL.

Total 17: horse 5; sheep 3; hare 2; wild pig 2; cattle 1; fallow deer 1; goat 1 ; mouflon 1 ; rabbit 1

\section{Sordaria humana (Fuckel) G. Winter}

1) Grosseto, Principina terra, 42d 43' 14.8146" 11d 1' 42.189", 0 m, on horse dung in d.c., F. Doveri, 2.4.98, CLSM 03495 penta. 2) Grosseto, Principina terra, 42d 43' 14.8146" 11d 1' 42.189", $0 \mathrm{~m}$, on rabbit dung in d.c., F. Doveri, CLSM 03495 esa.

Total 2: horse 1; rabbit 1

\section{Sordaria lappae Potebnia}

1) Livorno, Montioni, 43d 1' 57.7086" 10d 45' 1.674", $50 \mathrm{~m}$, on rabbit dung in d.c., F. Doveri, 18.3.09, CLSM 059.00 quater. 2) Livorno, Elba island, Campo nell'Elba, Pradazzo-Valle Buia, 42d 44' 41.64" 10d 10' 15.3732", 200 m, on hare dung in d.c., C. Cotta, 5.9.11, CLSM 
059.00-X. 3) Livorno, Elba island, ?, ? m, on sheep dung in d.c., L. Levorato, 9.4.11, CLSM 059.00-XI. 4) Livorno, Elba island, ?, ? m, on rabbit dung in d.c., L. Levorato, 9.4.11, CLSM 059.00-XII. 5) Livorno, Quercianella, 43d 27' 49.521" 10d 22' 47.7654", $0 \mathrm{~m}$, on fallow deer dung, F. Doveri, 19.12.12, CLSM 059.00-XIV. 6) Livorno, Quercianella, 43d 27' 49.521" 10d 22' 47.7654", 0 m, on fallow deer dung in d.c., F. Doveri, 23.12.12, CLSM 059.00-XV. 7) Livorno, Quercianella, 43d 27' 49.521" 10d 22' 47.7654", 0 m, on wild pig dung in d.c., F. Doveri, 31.5.13, CLSM 059.00-XVI. 8) Livorno, Quercianella, 43d 27' 49.521" 10d 22' 47.7654", 50 m, on wild pig dung in d.c., F. Doveri, 31.5.13, CLSM 059.00-XVII. 9) Livorno, Elba island, Campo nell'Elba, Pradazzo-Valle Buia, 42d 44' 41.64" 10d 10' 15.3732", 200 m, on hare dung in d.c., A. Pierulivo, 10.8.13, CLSM 059.00-XVIII.

Total 9: fallow deer 2; hare 2; rabbit 2; wild pig 2; sheep 1

Sordaria superba De Not.

1) Grosseto, Follonica, 42d 55' 59.6568" 10d 43' 41.6454", 0 m, on fallow deer dung in d.c., F. Doveri, 8.7.05, CLSM 03197-XXV. 2) Livorno, Elba island, Campo nell'Elba, Pradazzo-Valle Buia, 42d 44' 41.64" 10d 10' 15.3732", 200 m, on hare dung in d.c., C. Cotta, 5.9.11, CLSM 03197XXIX. 3) Livorno, Elba island, ?, ? m, on sheep dung in d.c., L. Levorato, 9.4.11, CLSM 03197XXX. 4) Livorno, Elba island, ?, ? m, on rabbit dung in d.c., L. Levorato, 9.4.11, CLSM 03197XXXI.

Total 4: fallow deer 1; hare 1; abbit 1; sheep 1

Sporormia fimetaria (De Not.) De Not.

Grosseto, Giglio island, Castello, 42d 21' 58.608" 10d 54' 1.7886", 400 m, on wild rabbit dung in d.c., S. Rum, 22.3.12, CLSM 03497 quater.

Sporormiella australis (Speg.) S.I. Ahmed \& Cain

1) Livorno, Elba island, Campo nell'Elba, Pradazzo-Valle Buia, 42d 44' 41.64" 10d 10' 15.3732", 200 m, on mouflon dung in d.c., C. Cotta, 5.10.10, CLSM 03197-XXIX. 2) Grosseto, Giglio island, Castello, 42d 21' 58.608" 10d 54' 1.7886", $400 \mathrm{~m}$, on wild rabbit dung in d.c., S. Rum, 22.3.12, CLSM 01597-XXXII.

Total 2: mouflon 1; wild rabbit 1

Sporormiella grandispora (Speg.) S.I. Ahmed \& Cain

Pisa, S. Rossore Park, 43d 43' 18.2418" 10d 17' 55.1502", 0 m, on fallow deer dung, F. Doveri, 5.3.00, CLSM 006.00 bis.

Sporormiella intermedia (Auersw.) S.I. Ahmed \& Cain

1) Pisa, S. Rossore Park, 43d 43' 18.2418" 10d 17' 55.1502", 0 m, on fallow deer dung in d.c., V. Caroti, 26.2.97, MCVE 588. 2) Pisa, S. Rossore Park, 43d 43' 18.2418" 10d 17' 55.1502", 0 $\mathrm{m}$, on fallow deer dung, F. Doveri, 5.3.00, CLSM 00597-XVI. 3) Pisa, S. Rossore Park, 43d 43' 18.2418" 10d 17' 55.1502", 0 m, on wild rabbit dung in d.c., F. Doveri, 5.3.00, CLSM 00597-XVII. 4) Livorno, Corbolone, 43d 33' 19.6272" 10d 22' 31.0764", $150 \mathrm{~m}$, on horse dung, G. Cacialli and F. Doveri, 14.4.00, CLSM 00597-XVIII. 5) Livorno, Elba island, Campo nell'Elba, Pradazzo-Valle Buia, 42d 44' 41.64" 10d 10' 15.3732", 200 m, on mouflon dung in d.c., C. Cotta, 5.10.10, CLSM 00597-LXX. 6) Livorno, Pianosa island, 42d 35' 3.501" 10d 4' 46.6068", 0 m, on wild rabbit dung in d.c., L. Levorato, 8.4.11, CLSM 00597-LXXII. 7) Grosseto, Giglio island, Castello, 42d 21' 58.608" 10d 54' 1.7886", 400 m, on wild rabbit dung, S. Rum, 22.3.12, CLSM 00597-LXXIII. 8) Grosseto, Montecristo island, 42d 20' 50.3298" 10d 19' 2.2404", ? m, on goat dung, J. Viacava, 10.5.13, CLSM 00597-LXXVII.

Total 8: wild rabbit 3; fallow deer 2; goat 1; horse 1; mouflon 1 
Sporormiella isomera

Livorno, Elba island, Campo nell'Elba, Pradazzo-Valle Buia, 42d 44' 41.64" 10d 10' 15.3732", 200 m, on hare dung in d.c., A. Pierulivo, 10.8.13, CLSM 009.13.

\section{Sporormiella lasiocarpa Lorenzo}

Grosseto, Giglio island, Castello, 42d 21' 58.608" 10d 54' 1.7886", 400 m, on wild rabbit dung, S. Rum, 22.3.12, CLSM 06698 ter.

Sporormiella minima (Auersw.) S.I. Ahmed \& Cain

1) Livorno, Palazzi di Cecina, 43d 19' 40.7532" 10d 29' 53.2422", 0 m, on cattle dung, F. Doveri, 30.4.06, CLSM 03595-LVIII. 2) Livorno, Suvereto, 43d 3' 50.3856" 10d 39' 31.6728", 0 m, on horse dung in d.c., F. Doveri, 18.3.09, CLSM 03595-LXIII. 3) Livorno, Montioni, 43d 1' 57.7086" 10d 45' 1.674", $50 \mathrm{~m}$, on rabbit dung in d.c., F. Doveri, 18.3.09, CLSM 03595 LXXI. 4) Lucca, Viareggio, 43d 52' 48.9462" 10d 13' 56.9166", 0 m, on horse dung, G. Cacialli, 30.3.13, CLSM 03595-LXXVII.

Total 4: horse 2; cattle 1; rabbit 1

\section{Sporormiella minimoides S.I. Ahmed \& Cain}

Livorno, Elba island, Campo nell'Elba, Pradazzo-Valle Buia, 42d 44' 41.64" 10d 10' 15.3732", 200 m, on hare dung in d.c., C. Cotta, 5.9.11, CLSM 01497 quater.

Sporormiella teretispora S.I. Ahmed \& Cain

Lucca, Viareggio, 43d 52' 48.9462" 10d 13' 56.9166", 0 m, on horse dung, G. Cacialli, 30.3.13, CLSM 010.02-ter.

Trichodelitschia minuta (Fuckel) N. Lundq.

Grosseto, Giglio island, Castello, 42d 21' 58.608" 10d 54' 1.7886", 400 m, on wild rabbit dung in d.c., S. Rum, 22.3.12, CLSM 04396-XVIII.

Tripterosporella heterospora (Mukerji, R.N. Kumar \& N. Singh) Doveri var. heterospora

Livorno, Suvereto, 43d 3' 50.3856" 10d 39' 31.6728", 0 m, on horse dung in d.c., F. Doveri, 18.3.09, CLSM 007.09.

Westerdykella cylindrica (Malloch \& Cain) Arx

Lucca, Viareggio, 43d 52' 48.9462" 10d 13' 56.9166", 0 m, on horse dung, G. Cacialli, 30.3.13, CLSM 005.13.

Zopfiella erostrata (Griffiths) Udagawa \& Furuya

Livorno, Montioni, 43d 1' 57.7086" 10d 45' 1.674", 0 m, on horse dung in d.c., F. Doveri, 18.3.09, CLSM 005.02 quater.

Zopfiella longicaudata (Cain) Arx

1) Pisa, Calambrone stables, 43d 36' 20.4876" 10d 18' 48.6036", 0 m, on horse dung in d.c., F. Doveri, 6.95, MCVE 482. 2) Livorno, Suvereto, 43d 3' 50.3856" 10d 39' 31.6728", 0 m, on horse dung, F. Doveri, 18.3.09, CLSM 01895-XVII.

Total 2: horse 2 


\section{Taxonomy}

Lophotrichus macrosporus (Faurel \& Locq.-Lin.) Arx et al., Beih. Nova Hedw. 94: 271988.

$\equiv$ Kernia macrospora Faurel \& Locq.-Lin., Rev. Mycol. 41: 5191977.

Figs 1-5

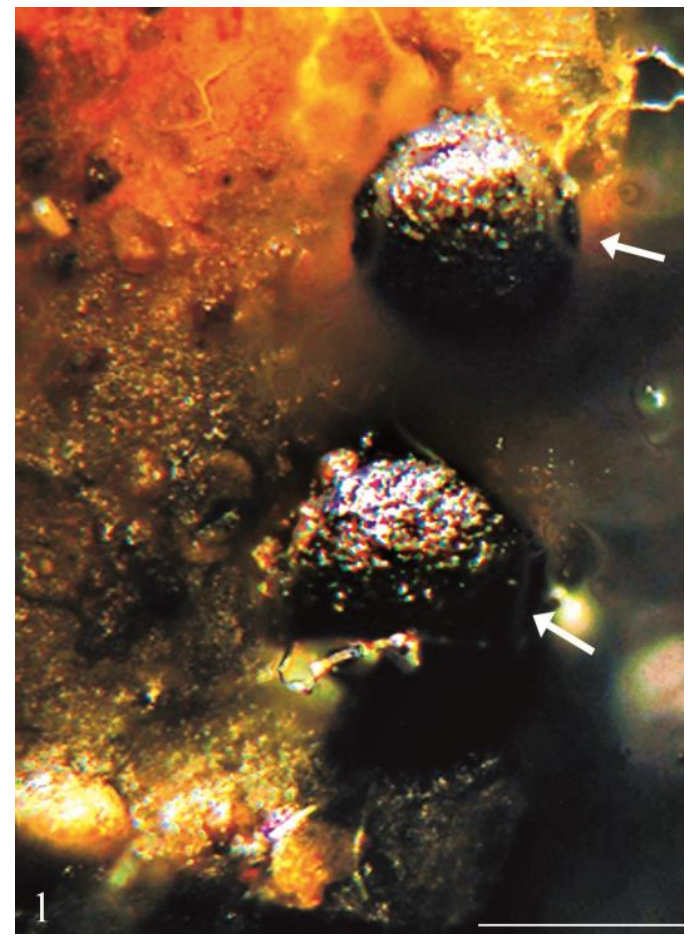

Fig. 1 - Lophotrichus macrosporus. Ascomata (arrows) on dung. - Bar $=250 \mu \mathrm{m}$.

Material examined - Livorno, Elba island, ?, ? m a.s.l., one superficial specimen on sheep (Ovis aries) dung in d.c., 9 Apr 2011, L. Levorato, CLSM 004.12; Livorno, Capraia island, 43d 2' 6" 9d 50' 36", 0 m a.s.l., on hedgehog (Erinaceus Europaeus) dung in d.c., 11 Apr. 2011, L. Levorato, CLSM 004.12 bis.

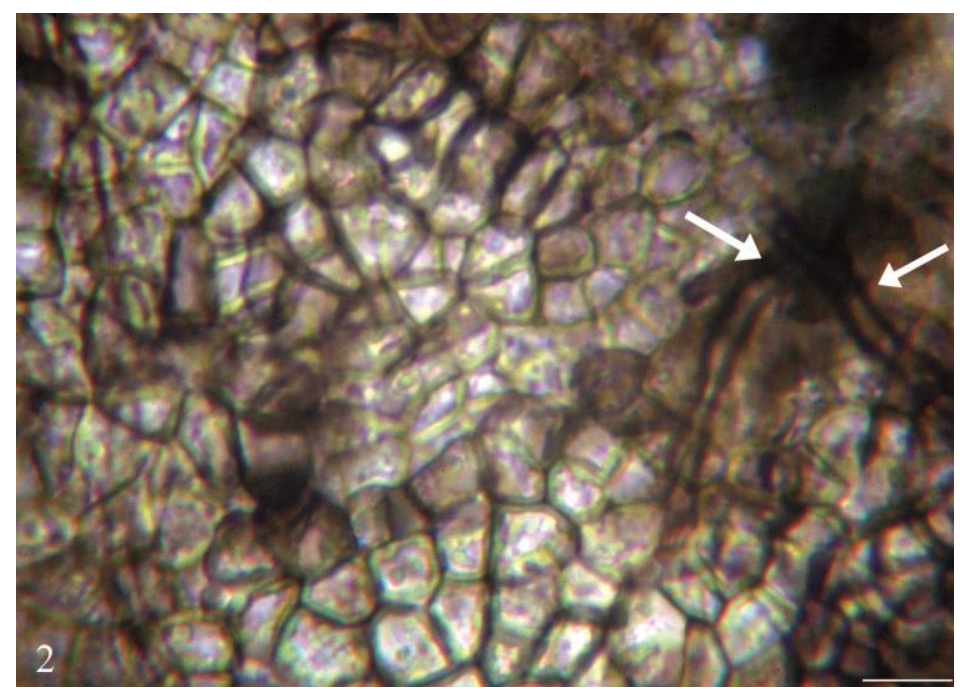

Fig. 2 - Lophotrichus macrosporus. Detail of excipulum with bases (arrows) of hyphoid hairs. Bar $=10 \mu \mathrm{m}$. 
Ascomata cleistothecioid, globose, 180-250 $\mu \mathrm{m}$ diam., blackish, semi-membranous, seemingly glabrous. Peridium some layers of very thick-walled, polygonal cells (textura angularis), 5-9 $\times 4-8 \mu \mathrm{m}$, the outermost dark brown, the innermost paler, thin-walled, up to $18 \times 15 \mu \mathrm{m}$. Some dark grey, septate, hyphoid hairs, 1-3 $\mu \mathrm{m}$ diam. arise from the outer cells. Paraphyses not seen. Asci unitunicate, non-amyloid, broadly clavate, ephemeral, thin-walled, 8-spored, 40-45 $\times 18-22 \mu \mathrm{m}$, with a short stalk. Ascospores (11-) 12-13 (-14) $\times 8-9(-9.5) \mu \mathrm{m}(\mathrm{Q}=1.26-1.62$; average $\mathrm{Q}=$ 1.47), irregularly biseriate to conglobate, dextrinoid in the early stages, ellipsoidal with rounded ends, fairly thick-walled, hyaline at first, becoming yellowish, finally straw-coloured to olivebrown, with subacute ends and two germ pores. Asexual state not observed.

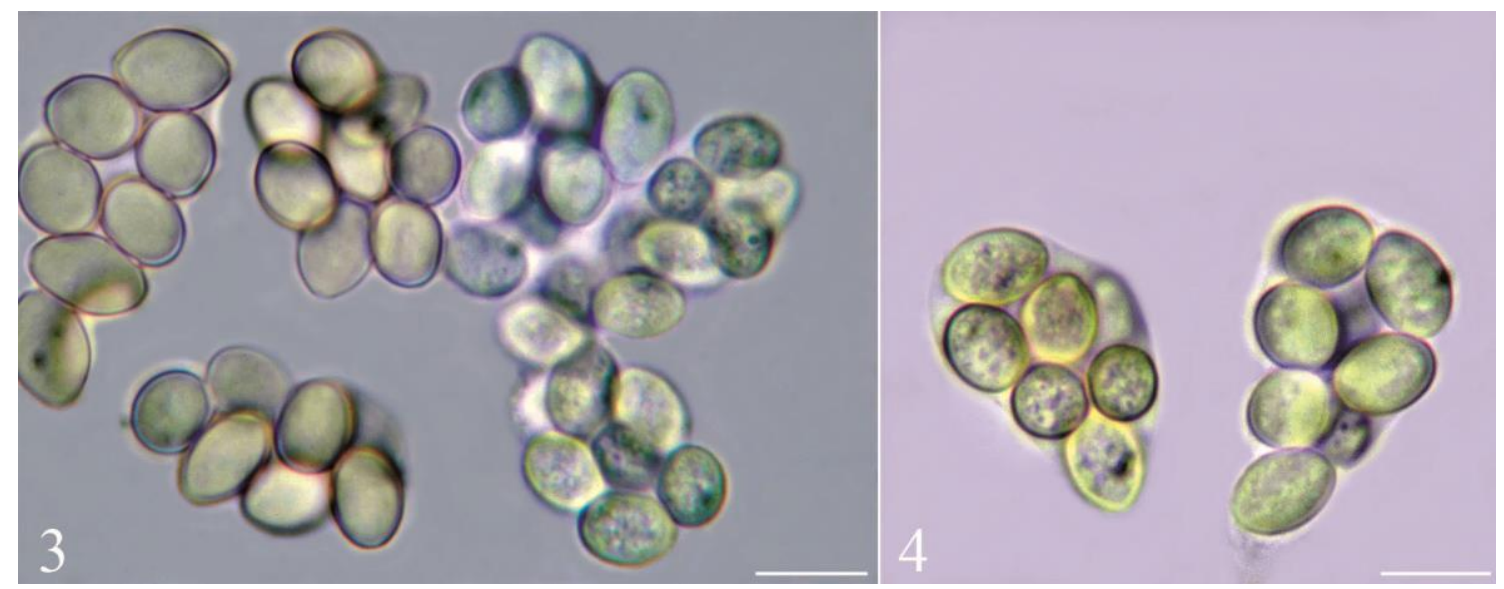

Figs 3-4 - Lophotrichus macrosporus. 8-spored asci with ascospores in different stages. - Bars $=$ $13 \mu \mathrm{m}$.

Notes - Lophotrichus R.K. Benj., in Microascaceae Luttr. ex Malloch (Microascales Luttr. ex Benny \& R.K. Benj.) encompasses seven cellulolytic species (Guarro et al. 2012) characterized by growth on dung or other media containing cellulose, dark, globose to ampulliform, membranous, very small, sometimes hairy perithecia (often beaked) or cleistothecia with a peridium of textura angularis, and no or Graphium-like asexual state. Periphysoids and paraphyses are absent, the ephemeral, globose, broadly clavate or ovoid, catenate or irregularly disposed asci lack an apical ring, and dextrinoid, one-celled, ellipsoidal to broadly fusiform or geniculate, reddish- or olivebrown ascospores have two germ pores (Malloch 1970, von Arx et al. 1988, Guarro et al. 2012).

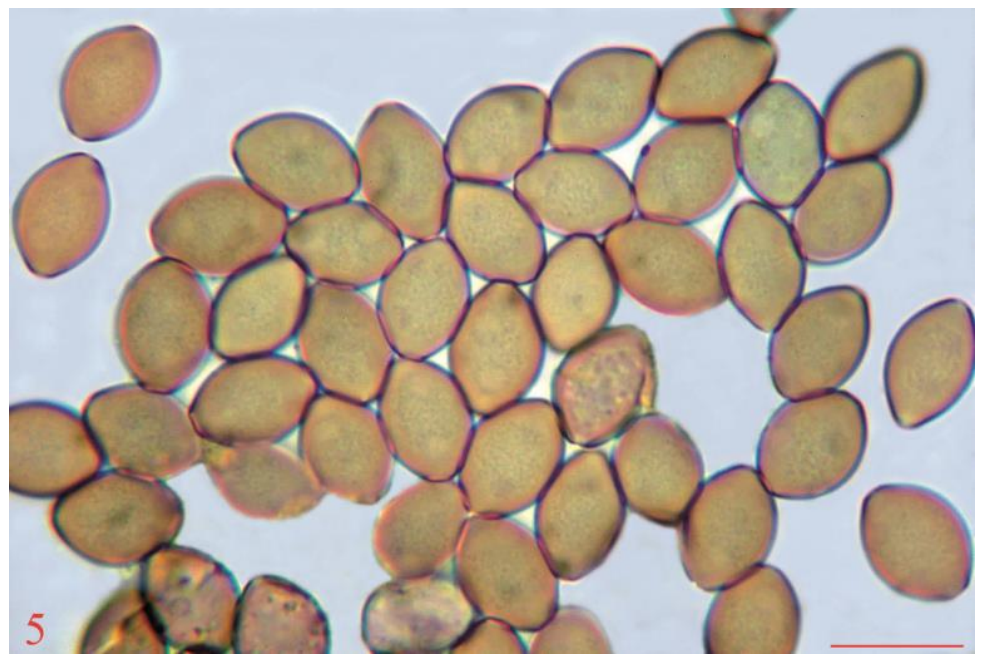

Fig. 5 - Lophotrichus macrosporus. Mature ascospores. - Bar $=13 \mu \mathrm{m}$. 
Lophotrichus is morphologically related to Microascus Zukal and Pseudallescheria Negr. \& I. Fisch., but Microascus has smaller, usually asymmetrical and inconspicuously uniporate ascospores, and Cephalotrichum Link, Scopulariopsis Bainier, Wardomyces F.T. Brooks \& Hansf. and Wardomycopsis Udagawa \& Furuya asexual states (von Arx et al. 1988, Kirk et al. 2008, Guarro et al. 2012), whereas Pseudallescheria has always non-ostiolate ascomata, an exoperidium of textura epidermoidea and, besides Graphium Corda, a Scedosporium Sacc. ex Castell. \& Chalm. asexual state (McGinnis et al. 1982, von Arx et al. 1988, Guarro et al. 2012).

Phylogenetic studies (Issakainen 1999, 2003, Rainer \& de Hoog 2006) proved Pseudallescheria fimeti (Arx et al.) McGinnis et al. not to be strictly related to the other Pseudallescheria spp., and suggested it to belong to another genus in the same family. A comparative, morphological and molecular study (Gilgado et al. 2007) also proved P. fimeti to nest with two Lophotrichus species, far from species of Pseudallescheria and Petriella, and the type strain to be characterised by cleistothecia with a peridium of textura angularis, not of a textura epidermoidea as described in the protologue (von Arx 1978). The results of these studies justified the recombination of Pseudallescheria fimeti in Lophotrichus fimeti (Arx et al.) Gilgado et al.

Lophotrichus macrosporus is characterised by globose, glabrous (with some hyphoid hairs at most), non-ostiolate ascomata lacking a neck, comparatively large ascospores, absence of asexual state (Locquin-Linard 1977, von Arx et al. 1988), and growth on various types of herbivore dung (Locquin-Linard 1977, Valldosera et al. 1987, Valldosera 1991), occasionally on soil (Fort et al. 1990). Ascospores of our collections are very similar in size to the protologue (Locquin-Linard 1977) and some subsequent records (Valldosera et al. 1987, Valldosera 1991), whereas those measured by von Arx et al. (1988) and Guarro et al. (2012) on the type subcultures are somewhat longer and narrower on average $(13-16 \times 7-9 \mu \mathrm{m})$.

Lophotrichus macrosporus is morphologically similar to L. bartlettii (Massee \& E.S. Salmon) Malloch \& Cain and L. fimeti: L. bartlettii has not an asexual state (von Arx et al. 1988, Delgado Avila et al. 2001b) and grows on dung of several herbivores, like L. macrosporus, but it differs in having perithecioid, very short-necked to papillate, or even double-papillate (Pathak \& Agrawal 1974) ascomata with an inconspicuous ostiole crowned with long terminal hairs, and smaller $(5.5-10 \times 4.5-7 \mu \mathrm{m})$ ascospores (Massee \& Salmon 1901, Ames 1963, Asad \& Ahmad 1968, Ahmed et al. 1971, Seth 1971, Furuya \& Udagawa 1973, Pathak \& Agrawal 1974, Valldosera 1991, Wang 1992, Delgado Avila et al. 2001a, Doveri 2011, Ribeiro Melo et al. 2012). Only von Arx et al. (1988) and Guarro et al. (2012) described somewhat larger ascospores $(8-12 \times 6-7.5$ $\mu \mathrm{m}$ ), but not so large as those of $L$. macrosporus. Only Furuya \& Udagawa (1973) reported $L$. bartlettii with a conidial state.

Lophotrichus fimeti, of which only the type strain (von Arx 1978) from Nilgai dung is known, is hardly distinguishable from L. macrosporus, with which it shares the spore size (11-13 $\times$ $8-10 \mu \mathrm{m})$ and almost glabrous, cleistothecioid ascomata. The presence of a Graphium-like asexual state and somewhat larger asci in L. fimeti appear to be the sole differences.

Besides L. macrosporus, Enterocarpus grenotii Locq.-Lin. is the only Lophotrichus-like species isolated by me from dung in the Tuscan Archipelago and coast. Doveri et al. (1997) and Doveri $(2004,2011)$ gave descriptions of this species and reasons why it is not a synonym of $L$. bartlettii (von Arx 1981a, von Arx et al. 1988, Valldosera 1991, Guarro et al. 2012).

Pleospora ambigua (Berl. \& Bres.) Wehm. var. ambigua, Mycologia 43: 421951.

Figs 6-16 三Pyrenophora ambigua Berl. \& Bres., Annu. Soc. Alpinisti Trid. 14: 441889.

Material examined - Lucca, Viareggio, 43d 52' 48.9462" 10d 13' 56.9166", 0 m a.s.1., two specimens strictly linked to a blade of grass on horse (Equus caballus) dung collected from coastal dunes, 30 Mar 2013, G. Cacialli, CLSM 006.13.

Pseudothecia perithecioid, pyriform, 250-280 × 150-180 $\mu \mathrm{m}$, semi-membranous, dark brown to blackish-brown, covered all over with sparse dark setae, with a setose, cylindrical neck, 

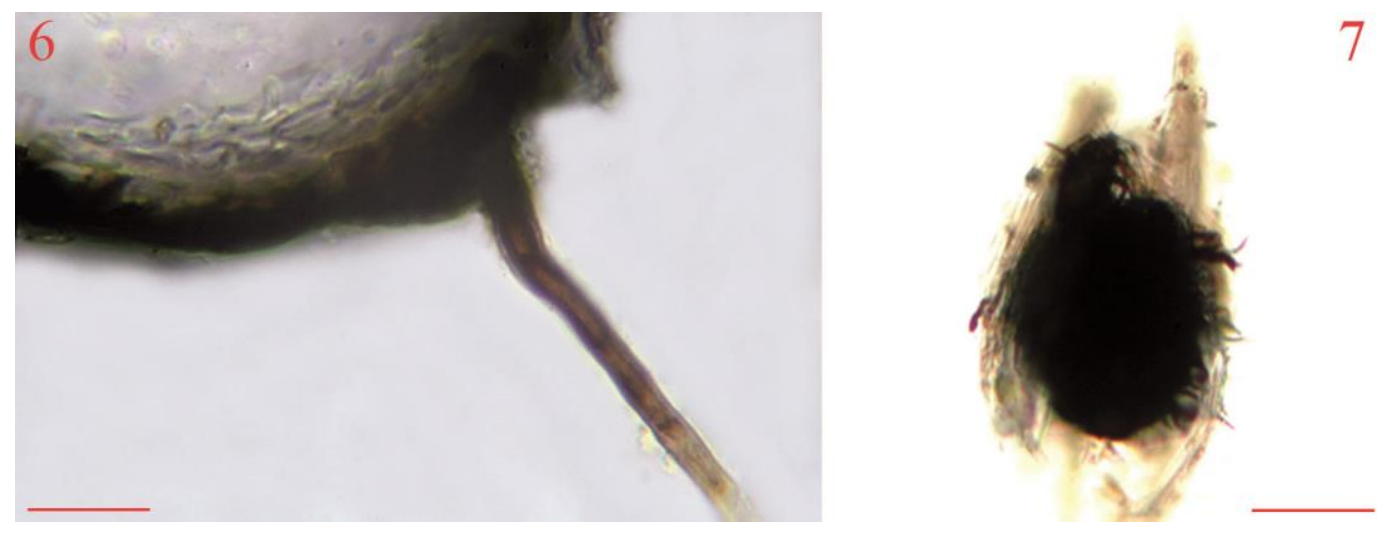

Figs 6-7 - Pleospora ambigua. 6, Base of ascoma with an anchoring hypha. 7, Setose ascoma in water - Bars $6=20 \mu \mathrm{m} .7=100 \mu \mathrm{m}$.

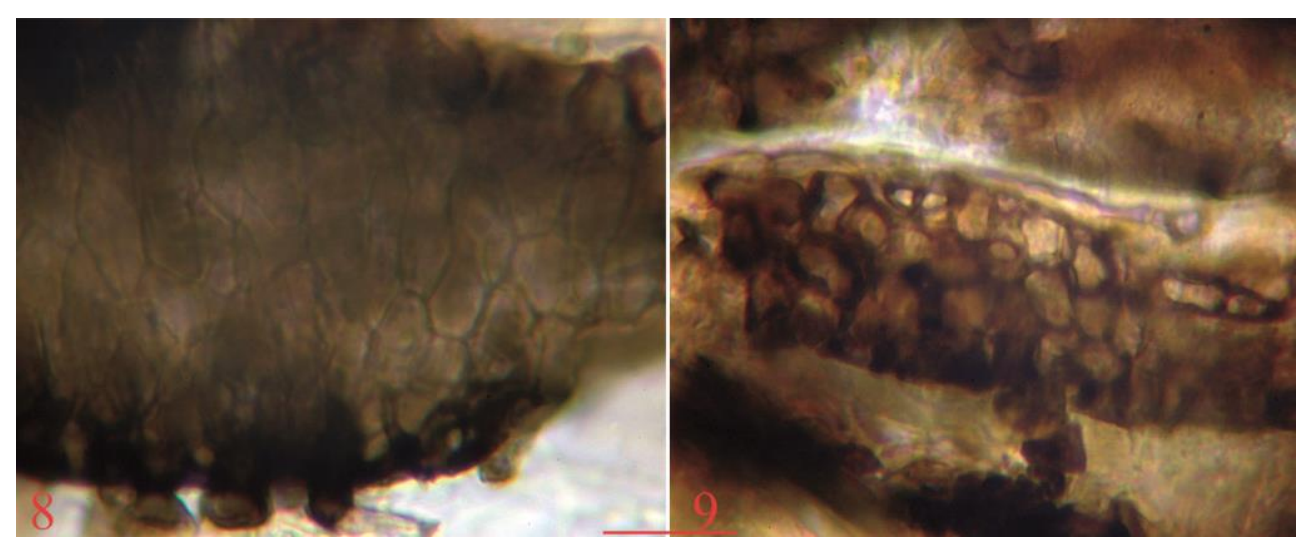

Figs 8-9-Pleospora ambigua. 8, Detail of endoperidium. 9, Detail of exoperidium - Bar $=20 \mu \mathrm{m}$.

up to $70 \times 40 \mu \mathrm{m}$. Dark, seta-like hyphe also present at the base, deeply anchoring to substrate. Peridium three-layered, pseudoparenchymatous: exoperidium thick, a textura angularis of dark brown, thick-walled, polygonal cells, 5-10 $\mu \mathrm{m}$ diam., a textura prismatica in the neck; mesoperidium of flattened, thin-walled, pale, subcylindrical cells; endoperidium of thin-walled, hyaline, polygonal cells. Setae flexuous to straight (on the neck), turned upwards, blackish-brown, paler in the upper portion, thick-walled, 40-60 $\times 4-5 \mu \mathrm{m}$ diam., up to $7 \mu \mathrm{m}$ diam. at the base, slightly pointed or somewhat rounded at the apex. Pseudoparaphyses mixed with asci and exceeding them, numerous, filiform, flexuous, sometimes branching, 1.5-2.5 $\mu \mathrm{m}$ diam., slightly enlarged at the apex, containing abundant hyaline vacuoles. Asci 90-110 × 15-20 $\mu \mathrm{m}, 8$-spored, non-amyloid, fissitunicate, cylindric to cylindric-clavate, rounded at the apex, with a short, lobate stalk. Ascospores uni- or biseriate, hyaline at first, becoming deep yellow, yellowish brown at maturity, (18-) 19-24 (-26) × 9-12 (-13) $\mu \mathrm{m}(\mathrm{Q}=1.76-2.26$; average $\mathrm{Q}=2.04)$, ellipsoidal, rarely somewhat longitudinally asymmetric, more rarely subclavate, smooth, lacking both germ pores and slits, thick-walled, usually roundish at the ends, sometimes slightly pointed at the lower end, muriform (dictyospores), subdivided into several cells by 5-7 (usually 6, exceptionally 7) transverse septa and 1 longitudinal septum, somewhat constricted at the third tranverse septum, with the upper half usually somewhat wider than the lower one, seemingly not surrounded by a gelatinous sheath. The uppermost cell with a quite flattened, rarely slightly angular base, often subdivided by the longitudinal septum; the lowermost cell not subdivided, usually angular. Asexual state: Stemphylium-like: only one conidium observed, dark-brown, muriform, globose, thick-walled, verrucose, slightly constricted in the middle, $14 \mu \mathrm{m}$ diam. 


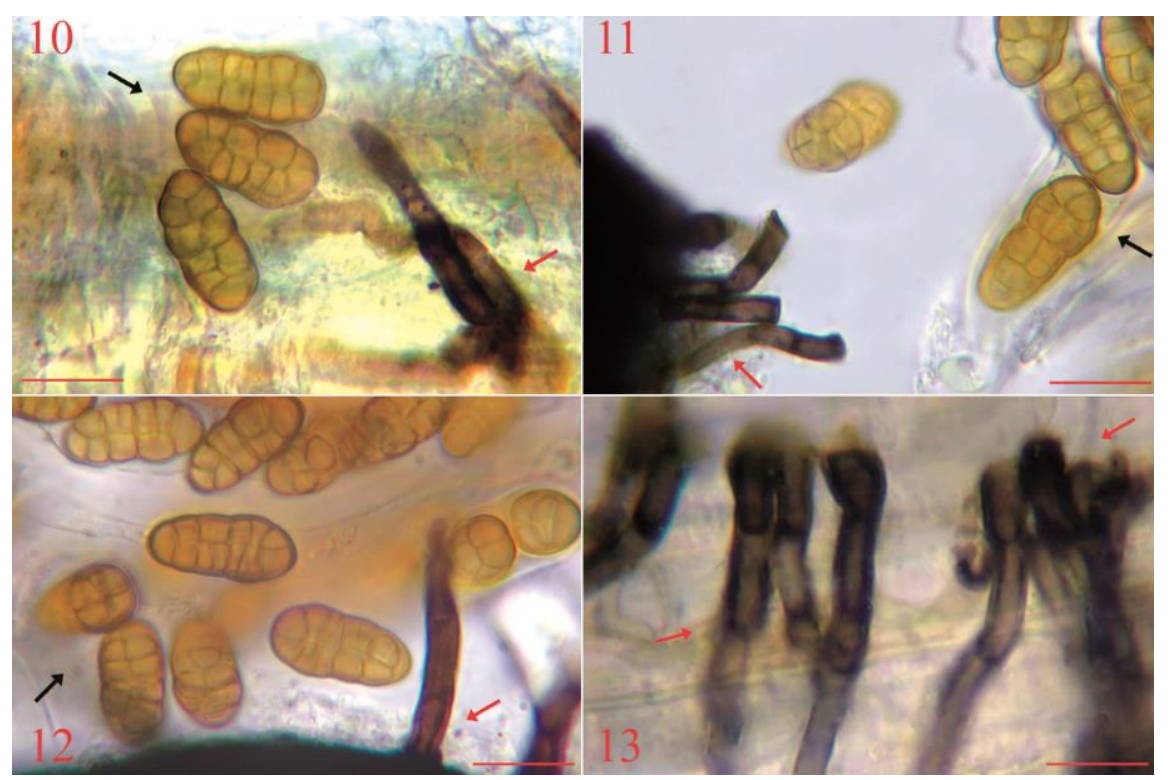

Figs 10-13 - Pleospora ambigua. Setae (red arrows) from different parts of pseudothecium and ascospores (black arrows) - Bars $=15 \mu \mathrm{m}$.

Notes - We briefly described the main morphological features of Pleospora Rabenh. ex Ces. $\&$ De Not. (Pleosporaceae Nitscke) in our work on coprophilous fungi from Italy (Doveri 2004). We also described a collection of $P$. herbarum (Pers. : Fr.) Rabenh. from dung, remarking that species in this genus usually develop immersed in leaves or stems of herbaceous and woody plants (Wehmeyer 1951, Simmons 1952), behaving as saprobes, occasionally as parasites. Collections of Pleospora spp. from dung are exceptional and concern, besides $P$. herbarum (Richardson 2011), $P$. pallida Sacc. \& Speg. and P. malacospora Speg., recorded from Italy by Spegazzini $(1878,1879)$, P. rubicunda Niessl, recorded from Denmark by Munk (1957), and now also P. ambigua.

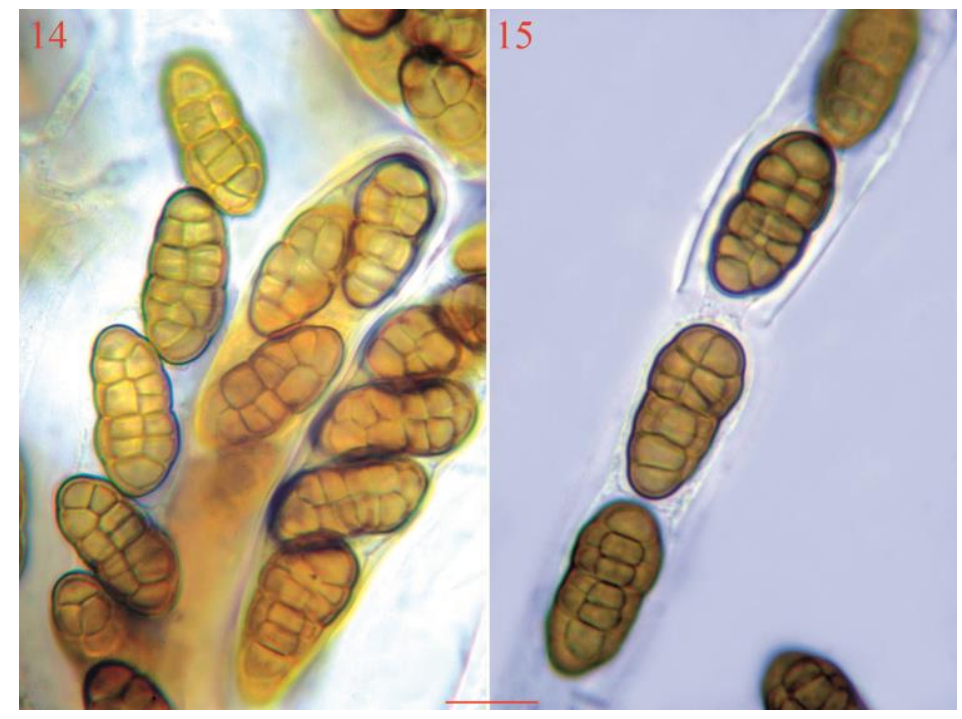

Figs 14-15 - Pleospora ambigua. Ascospores inside fissitunicate asci - Bar $=12 \mu \mathrm{m}$.

$P$. ambigua belongs to the group of $P$. herbarum, characterised by pseudothecia with a simple structure of the neck (periphyses absent), filiform, interascal pseudoparaphyses, muriform, deeply coloured ascospores often with additional septa in the end cells, no sclerotial tissue (Wehmeyer 1949, Munk 1957). 


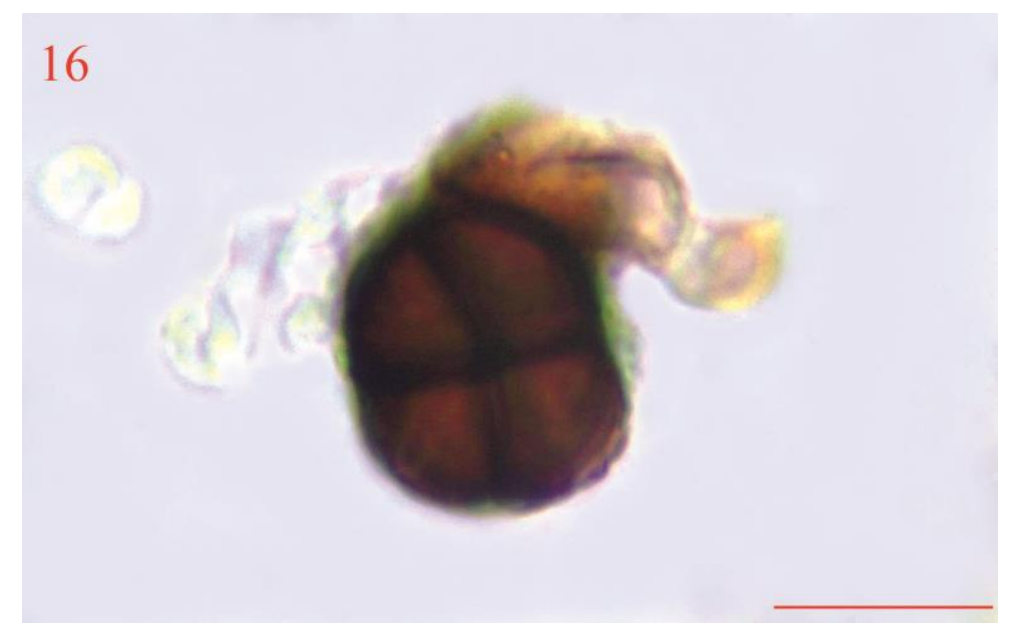

Fig 16 - Pleospora ambigua. Stemphylium-like anamorph - Bar $=10 \mu \mathrm{m}$.

Wehmeyer (1951) distinguished P. ambigua var. ambigua from P. ambigua var. crandallii (Ellis \& Everh.) Wehm., ascribing their macro- and microscopic differences to the habitat. The type variety grows on herbaceous stems, at lower altitudes, has larger, less hairy perithecia with both apical setae and seta-like basal hypae, and yellow-brown ascospores, whereas the var. crandallii preferably grows on leaves or small stems, at higher altitudes, has dark red-brown ascospores and dense setae only around the ostiole, and usually lacks basal hyphae (Ellis \& Everhart 1897). Our collection fully matches the type variety and collection described by Webster \& Lucas (1961).

Rhytidospora cainii Guarro, Mycologia 75: 9271983.

Figs 17-23

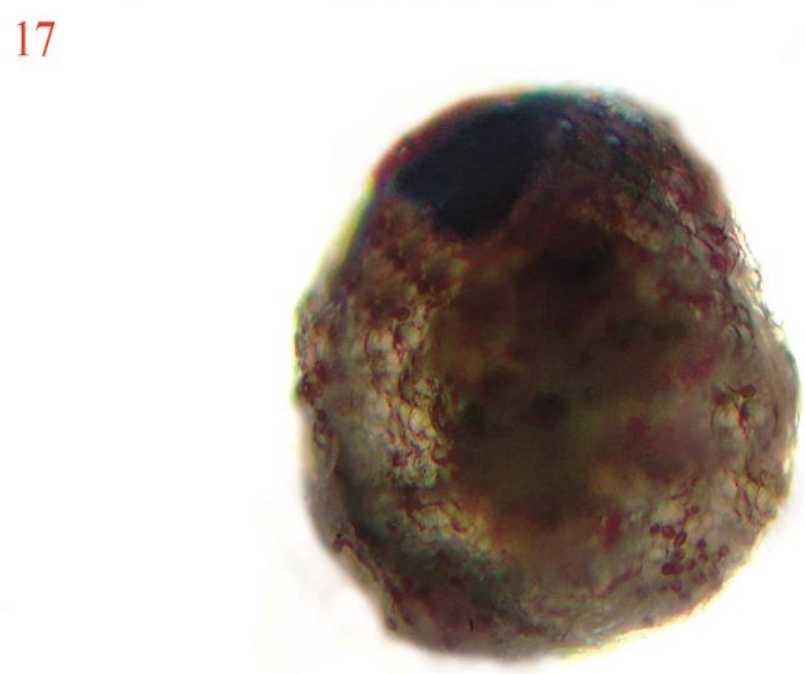

Fig 17 - Rhytidospora cainii. Ascoma in water - Bar $=75 \mu \mathrm{m}$.

Material examined - Livorno, Quercianella, 43d 27' 49.521" 10d 22' 47.7654", 50 m a.s.1, eight gregarious, superficial specimens on wild pig (Sus scrofa) dung and blotting paper at its base in d.c., 31 May 2013, F. Doveri, CLSM 007.13; Livorno, Quercianella, 43d 27' 49.521" 10d 22' 47.7654", 0 m, on wild pig dung in d.c., 31 May 2013, F. Doveri, CLSM 007.13 bis. 


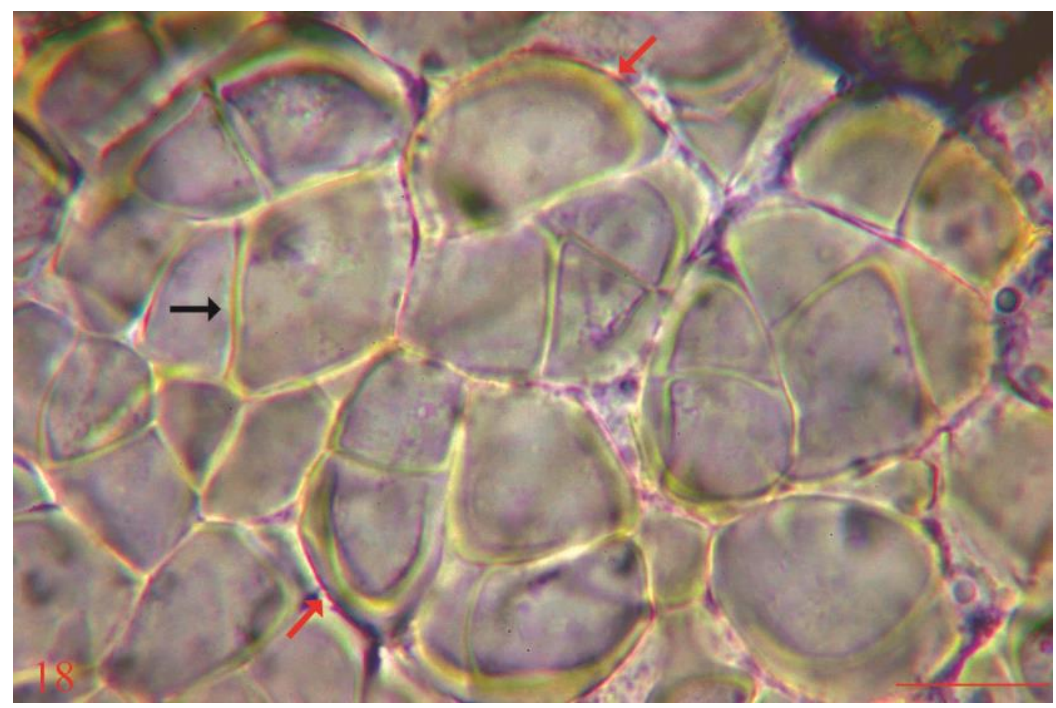

Fig 18 - Rhytidospora cainii. Plates of cephalothecoid peridium with thin inner walls (black arrow) and thick outer walls (red arrows) - Bar $=10 \mu \mathrm{m}$.

Ascomata cleistothecioid, globose, membraneous, rugulose, pale to deep yellow, translucent, sparsely hairy, 240-350 $\mu \mathrm{m}$ diam., darkening with age due to the maturing ascospores. Peridium thin, seemingly one-layered, indistinctly cephalothecoid as lacking radiating cells, formed of translucent plates of yellow polygonal cells, $12-25 \times 10-20 \mu \mathrm{m}$, thin-walled inside, but strongly thick-walled at the periphery of each plate, so marking out a dehiscence line. Hairs hardly observable at reflected light, flexuous, smooth, pale yellow, septate, thin-walled, 1.5-4 $\mu \mathrm{m}$ diam., up to $8 \mu \mathrm{m}$ enlarged at their base, usually slightly rounded at the apex. Paraphyses absent. Asci irregularly disposed, ephemeral, unitunicate, non-amyloid, 4-spored, subglobose to broadly ellipsoidal, 16-20 × 13-15 $\mu \mathrm{m}$, thin-walled, lacking an apical apparatus. Ascospores one-celled, hyaline and with one or two large drops in the early stages, becoming dark brown, thick-walled, wrinkled, indistinctly pitted at high magnification $(1000 \times)$, ellipsoidal, 11-13 $(-13.5) \times(6.5-) 7.5-$ $10 \mu \mathrm{m}, \mathrm{Q}=1.30-1.66$ (average $\mathrm{Q}=1.44$ ), often with truncate ends, with a prominent germ pore at each end, 1.5-2 $\mu \mathrm{m}$ diam. Asexual state not seen.

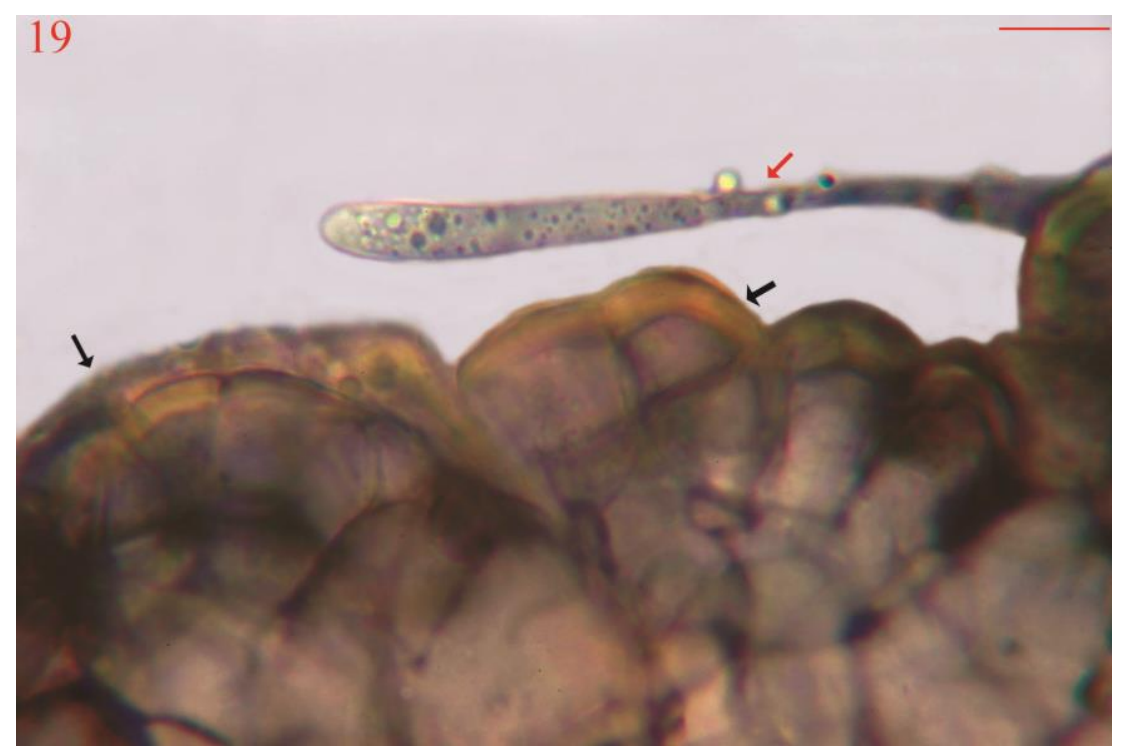

Fig 19 - Rhytidospora cainii. Peridial hair (red arrow) and plates with very thick outer walls (black arrows) - Bar $=10 \mu \mathrm{m}$. 
Notes - Rhytidospora Jeng \& Cain belongs to Melanosporales N. Zhang \& M. Blackw., an order phylogenetically close to Coronophorales Nannf. (Hibbett et al. 2007, Lumbsch \& Huhndorf 2007), encompassing the sole family Ceratostomataceae.

Ceratostomataceae G. Winter are characterised by non-stromatic, translucent, perithecial or cleistothecial ascomata, a centrum of evanescent, unitunicate, globose to clavate asci without apical apparatus, absence of interascal tissue, one-celled, dark coloured ascospores usually with a germ pore at each end, an hyphomycetous asexual state, and often a mycoparasitic behaviour (Barr 1990, Zhang et al. 2006, Cannon \& Kirk 2007).

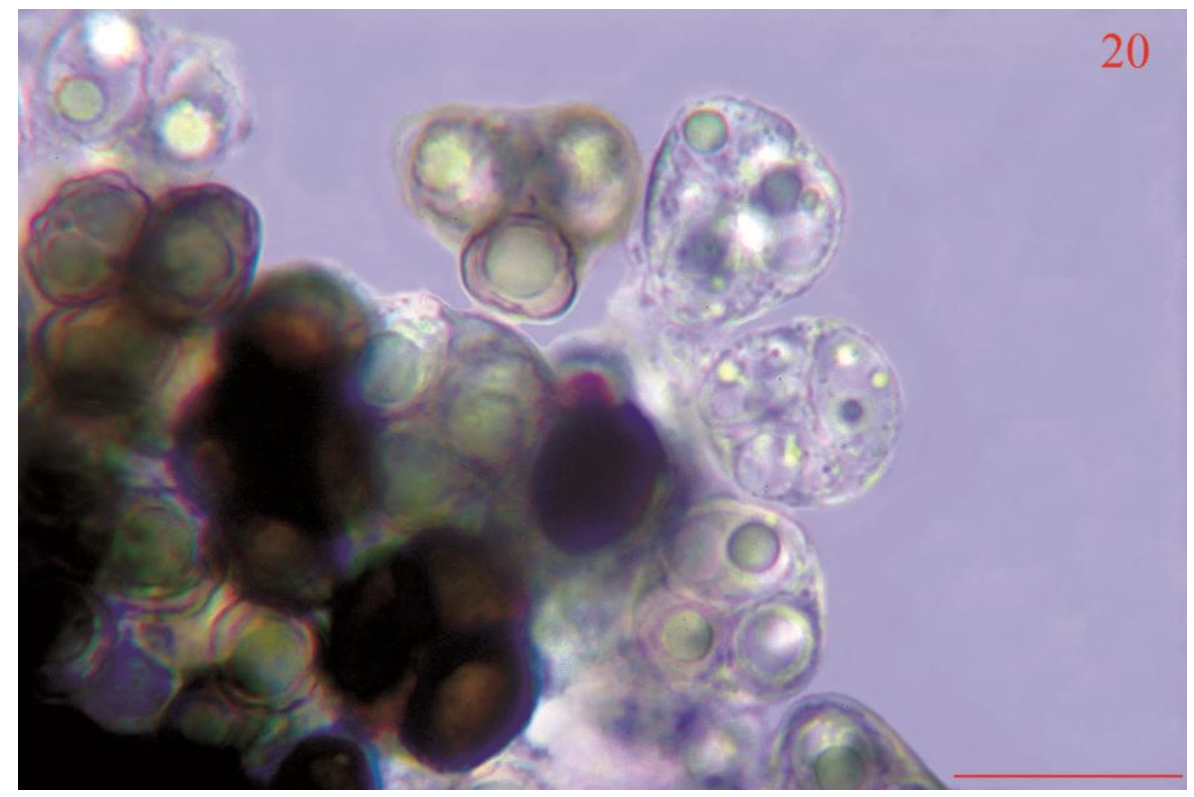

Fig 20 - Rhytidospora cainii. 4-spored asci in different stages - Bar $=20 \mu \mathrm{m}$.

Rhytidospora was erected by Jeng \& Cain (1977) to accomodate a single species, $R$. tetraspora Jeng \& Cain, isolated from burro dung, with most morphological features common to Melanosporaceae Bessey (= Ceratostomataceae), but with the peculiarity of wrinkled ascospores, a thick peridium with a cephalothecoid outer layer, and absence of asexual state. The exoperidial frame was particularly stressed, as consisting of typical, regular, petaloid plates, each with a central polygonal cell and peripherical, radiating cells with very thick outer walls, which draw an uninterrupted line of dehiscence (Jeng \& Cain 1977). R. tetraspora was later recorded from horse (Erksson 1999) and camel (Abdullah \& Alutby 1999) dung.

Krug \& Jeng (1979) erected $R$. bispora, a species isolated from cattle dung which, like $R$. tetraspora, has smooth, translucent, cleistothecial ascomata, but 2-spored asci, somewhat larger $(12-14 \times 7-10 \mu \mathrm{m})$ ascospores, and a different cephalothecoid peridium. Krug \& Jeng (1979) did not mention a layered peridium in $R$. bispora, so we can suppose that it is thinner in this species than in $R$. tetraspora. The cephalothecoid peridium of $R$. bispora is also simpler (Krug \& Jeng 1979), not petaloid, as the plates lack radiating cells and are formed of peripherically thick-walled, polygonal cells in clusters.

Valldosera et al. (1991), describing a collection of $R$. bispora from cattle dung in Spain, stated that its ascospores are wrinkled but also pitted (both at light and electron microscopy), a feature not mentioned in the protologue (Krug \& Jeng 1979). In agreement with Guarro (1983) they supposed that pitted ascospores are typical of this genus, but unfortunately they forgot to explain if pitted ascospores are also present in the type material of $R$. bispora studied by them (Valldosera et al. 1991). Piontelli et al. (2006) recorded $R$. bispora from wild rabbit dung and noticed pitted ascospores in their two collections of this species. 


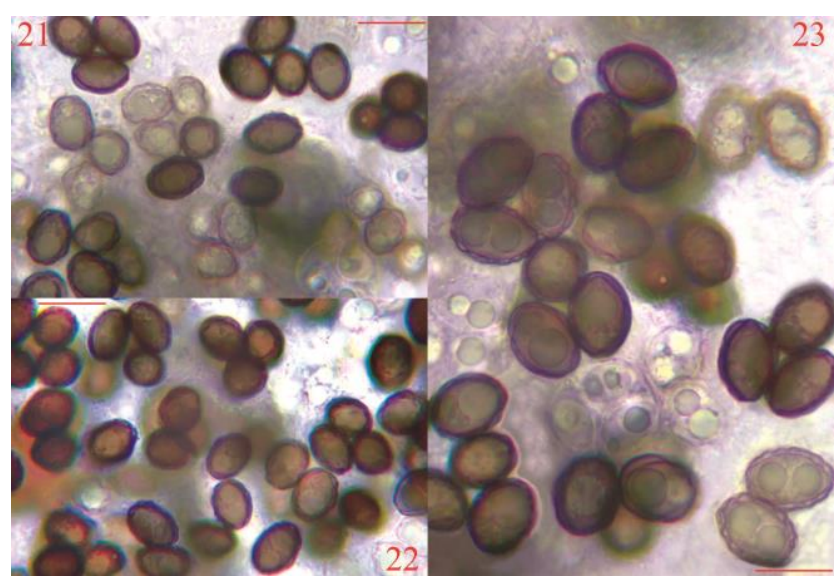

Figs 21-23 - Rhytidospora cainii. Ascospores - Bars 21-22 = $15 \mu \mathrm{m} .23=10 \mu \mathrm{m}$.

Krug \& Jeng (1979) also recombined Microthecium inordinatum Malloch \& Cain in Rhytidospora inordinata, a species originally described from cattle dung (Malloch \& Cain 1972) with 8-spored asci, wrinkled ascospores, and a two-layered peridium with an exostratum apparently of a texura angularis. Their study of the type material, however, revealed a simple cephalothecoid peridium, very similar to $R$. bispora.

$R$. cainii, originally recorded from sheep dung (Guarro 1983), is the first Rhytidospora sp. to be described with hairy cleistothecia and pitted, besides wrinkled, ascospores. It has, like $R$. tetraspora, 4-spored asci, but differs by a much simpler cephalothecoid peridium and slightly larger $(12-14 \times 8-10.5 \mu \mathrm{m})$ ascospores.

R. citriformis Valldos. \& Guarro is the latest new species described under Rhytidospora: isolated from cattle dung, characterised by glabrous and smooth cleistothecia with an epidermoid peridium, 2-spored asci, and warted, limoniform ascospores (Valldosera 1991, Valldosera \& Guarro 1994), it shows, in our opinion, many features not matching Rhytidospora, but a different genus in Ceratostomataceae. So we keep it out of the following world-key to Rhytidospora spp.:

1) Peridium thick, with a complex, cephalothecoid outer layer of radiating and central polygonal cells to form petaloid plates. Asci 4-spored. Ascospores 10-13 × 7-9 $\mu \mathrm{m}$ R. tetraspora

1*) Peridium thinner, simple cephalothecoid, with plates of sole polygonal cells 2

2) Ascospores 14.5-18.5 × 10.5-12.5 $\mu \mathrm{m}$. Asci 8-spored R. inordinata

2*) Ascospores smaller. Asci with fewer ascospores 3

3) Asci 2-spored. Ascospores 12-14 × 7-10 $\mu \mathrm{m}$ R. bispora

3*) Asci 4-spored. Ascospores 11-14 × 7.5-10.5 $\mu \mathrm{m}$ R. cainii

Sporormiella isomera S.I. Ahmed \& Cain, Canadian Journal of Botany 50: 445 1972. Figs 24-31

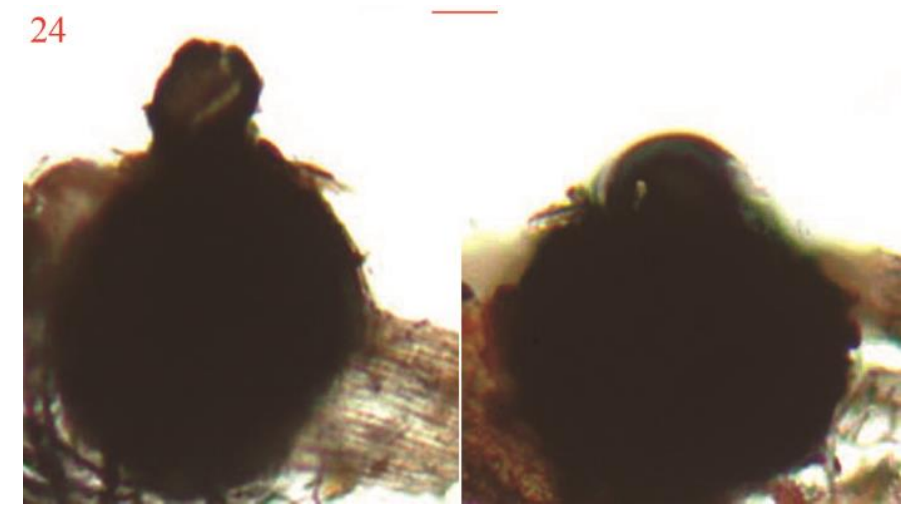

Fig 24 - Sporormiella isomera. Ascomata in water - Bar $=100 \mu \mathrm{m}$. 


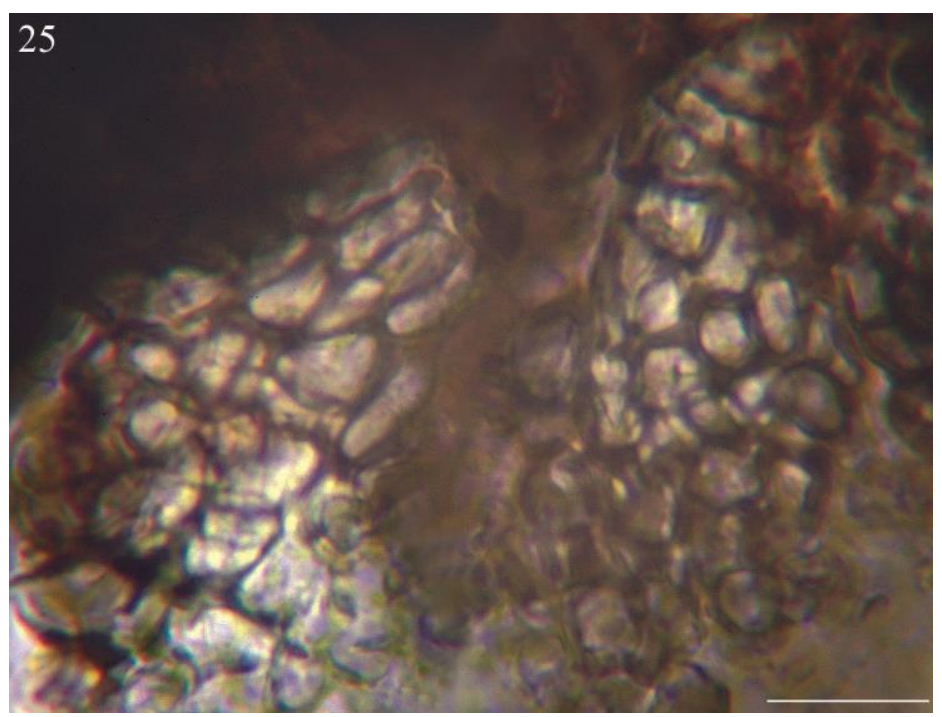

Fig 25 -Sporormiella isomera. Detail of exoperidium $-\mathrm{Bar}=20 \mu \mathrm{m}$.

Material examined - Livorno, Elba island, Campo nell'Elba, Pradazzo-Valle Buia, 42d 44' 41.64" 10d 10' 15.3732", $200 \mathrm{~m}$, about ten semi-immersed specimens on hare (Lepus europaeus) dung in d.c., 10 Aug 2013, A. Pierulivo, CLSM 009.13.

Pseudothecia subglobose to obpyriform, 220-270 $\mu \mathrm{m}$ diam., blackish, hairy in the lower part and at the base, membranous, with a subcylindric neck, 70-80 $\times$ 40-50 $\mu \mathrm{m}$. Peridium pseudoparenchymatous, two-layered: endostratum of pale brown, thin-walled, polygonal cells, 10 $13 \times 7-9 \mu \mathrm{m}$; exostratum of dark, thick-walled, polygonal cells, 5-8 $\times 4-6 \mu \mathrm{m}$, supporting numerous septate, branched, brown, hyphoid hairs. Pseudoparaphyses exceeding the asci, cylindricfiliform, 1-3 $\mu \mathrm{m}$ diam., septate, containing many hyaline vacuoles, often nodulose, branched (particularly at their bases) and sometimes anastomosed, not- or hardly inflated at the tips. Asci fissitunicate, non-amyloid, 8-spored, 115-150 × 13-15 $\mu \mathrm{m}$, cylindrical, lacking an apical apparatus, roundish at the apex, with a lobate, gently tapering, very short, 10-15 $\mu \mathrm{m}$ long stalk. Ascospores obliquely biseriate in the upper part of the ascus, uniseriate in the lower, hyaline at first, becoming yellowish, finally dark brown, 32-36 ×6-6.5 $\mu \mathrm{m}$, cylindric, smooth, thick-walled, surrounded by a broad gelatinous sheath which often bilaterally narrows at the septa, straight or slightly curved, 4celled, exceptionally 3-celled due to the anomalous lack of one septum, the cells easily separable at the central, fairly deep septum, the end cells conical with blunt apices, slightly longer than the middle and the uppermost cell often shorter than the lowermost, middle cells cylindrical and the second cell often somewhat wider than the others. Each cell with an oblique or subparallel, sigmoidal germ slit.

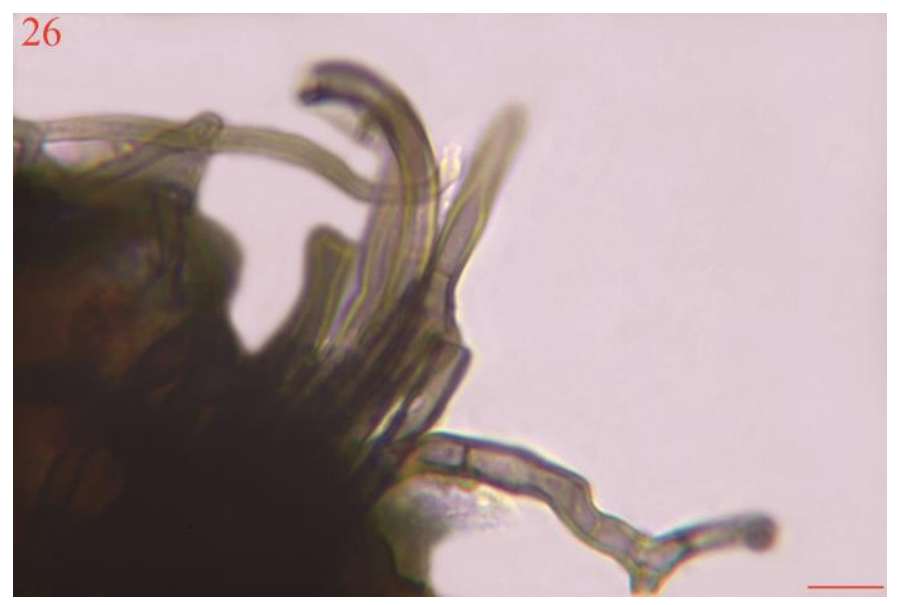

Fig 26 - Sporormiella isomera. Hyphoid hairs - Bar $=5 \mu \mathrm{m}$. 


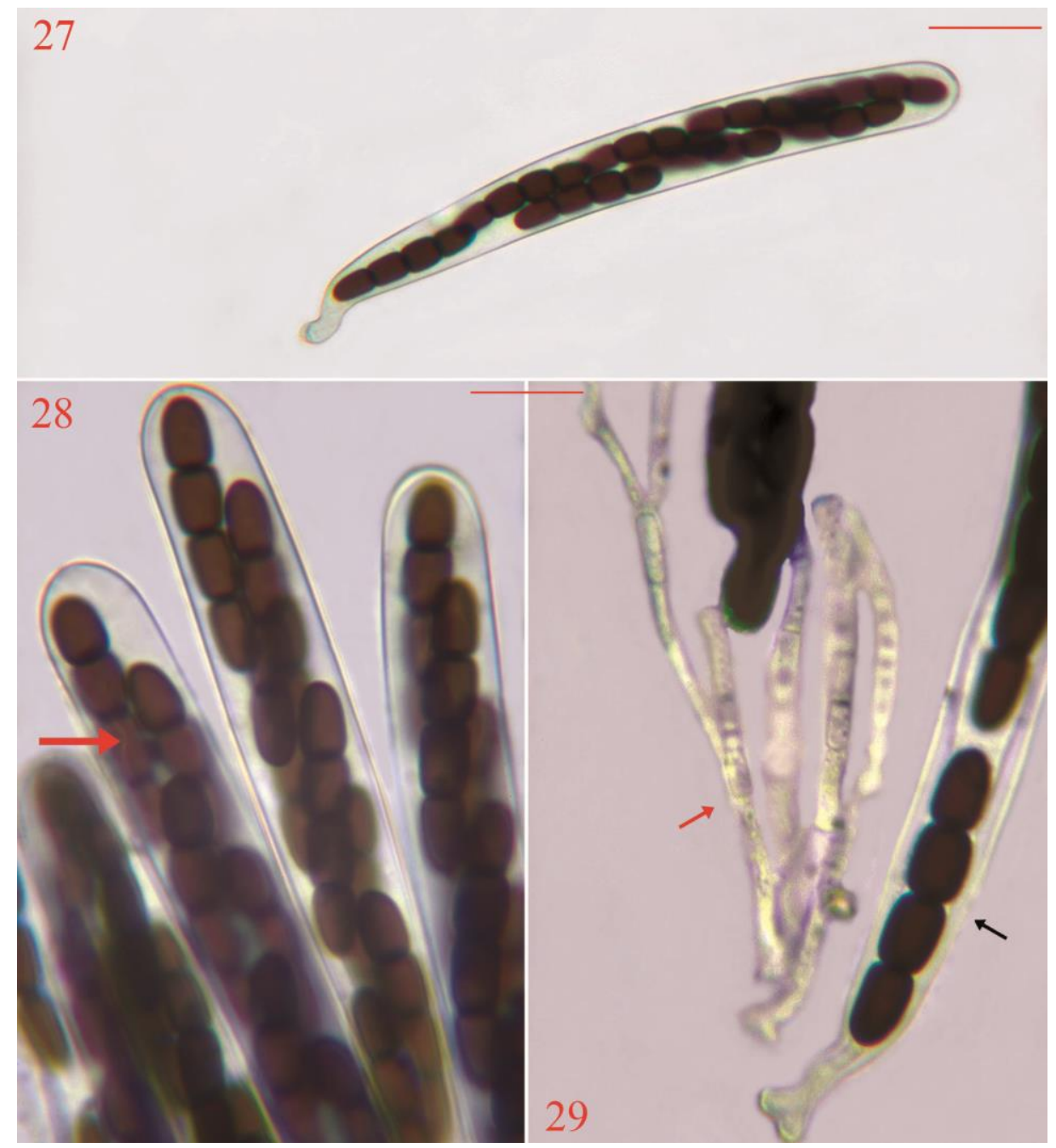

Figs 27-29 - Sporormiella isomera. 27, Short-stalked ascus with mature ascospores. 28, Upper part of asci with mature ascospores. A sigmoidal germ slit is observable (arrow). 29, Pseudoparaphyses (red arrow) and lower part of ascus (black arrow). - Bars $27=25 \mu \mathrm{m} .28=15 \mu \mathrm{m} .29=14 \mu \mathrm{m}$.

Notes - Ahmed \& Cain (1972) erected Sporormiella isomera as a species similar to $S$. leporina (Niessl) S.I. Ahmed \& Cain, but distinguishable by having asci with a shorter stipe (up to $15 \mu \mathrm{m}$ versus $20-35 \mu \mathrm{m}$ long), and ascospores with a less pointed upper end, sygmoidal, parallel to occasionally slightly oblique rather than oblique to diagonal germ slits, more separable cells, and absolutely transverse septa instead of tranverse septa, tending to oblique.

Doveri (2004) and Bell (2005) considered S. isomera as a possible later synonym of $S$. leporina as the differences stressed by Ahmed \& Cain (1972) are inconstant and several collections worldwide show intermediate features between them. After revising all my collections classified as S. leporina, I am changing my mind because I found that some differences cited by Ahmed \& Cain (1972) are actual and constant. In particular, I noticed that asci with significantly slenderer stalks are always associated to ascospores with a more pointed upper end-cell and non-sigmoidal germ slits (S. leporina). In contrast, asci with stocky, very short stalks are consistently associated with more rounded end spore cells, and sigmoidal, almost always parallel germ slits (S. isomera). The only difference from Ahmed \& Cain (1972) concerns the position of the germ slits, rather variable, parallel to diagonal in my collections of S. leporina. Taking into account the association of these differential characteristics, I reclassified my collections, and I concluded that only two out of eighteen are $S$. isomera, i.e. the collection described in this work and one from carivore dung. Except for a single collection from horse dung, all my other collections of S. leporina are from leporid and cervine dung. 


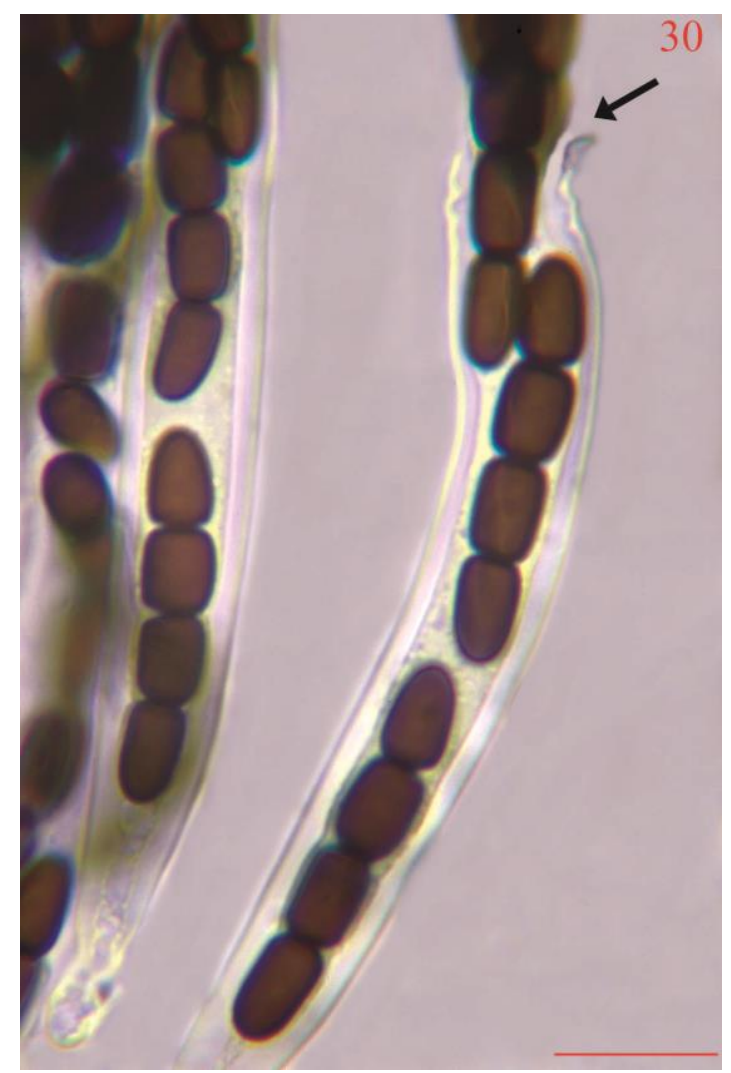

Fig 30 - Sporormiella isomera. Detail of fissitunicate (arrow) ascus - Bar $=15 \mu \mathrm{m}$.

Records of S. leporina throughout the world are in line with mine from Italy, mostly coming from leporine (Niessl 1878, Griffiths 1901, Cain 1934, Matsushima 1975, Valldosera \& Guarro 1990, Lundqvist 1997, Kruys \& Ericson 2008), cervine (Tóth 1967, Moyne \& Petit 2006, Richardson 2008, Lécuru 2013) and equine (Griffiths 1901, Wilson 1947, Parker 1979, Barrasa Gonzales 1985) dung, less frequently from dung of other herbivores (Ahmad \& Sultana 1973, Cribb 1994, Treigiené 2004, Welt \& Heine 2006), carnivores (Cain 1957, Ahmed \& Cain 1972), or even bird (Richardson 2004) dung. The few records worldwide of S. isomera (Minoura 1969, Ahmed \& Cain 1972, Furuya \& Udagawa 1972, Khan \& Cain 1979, Richardson 2004) mostly come from dung of various herbivores.

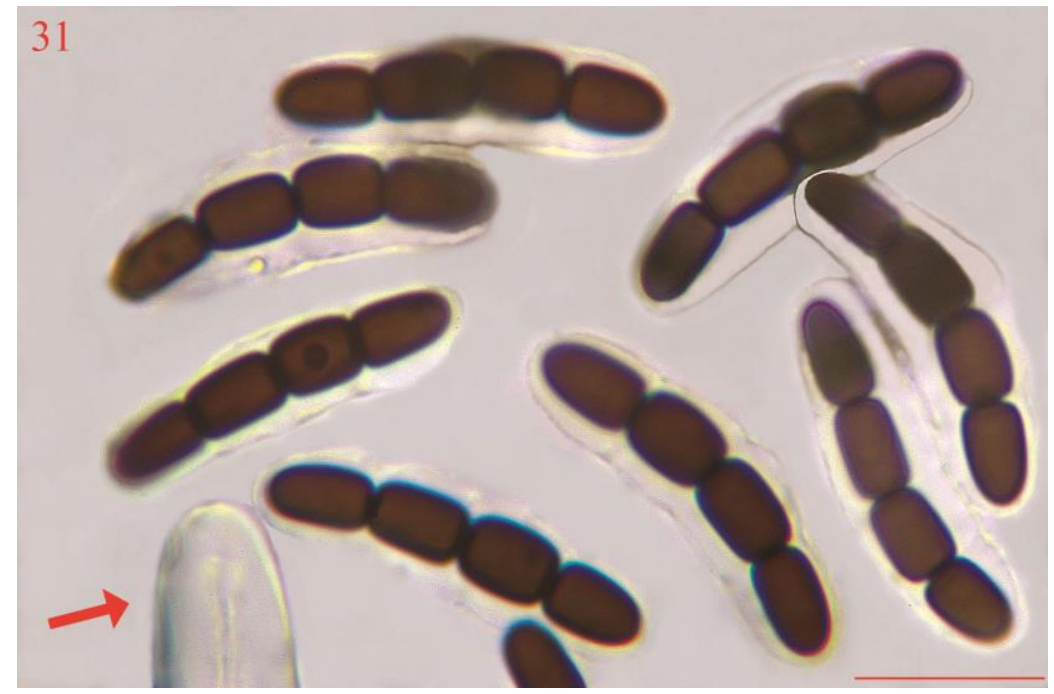

Fig 31 - Sporormiella isomera. Free ascospores and apex (arrow) of an immature ascus - Bar $=15$ $\mu \mathrm{m}$. 
Westerdykella cylindrica (Malloch \& Cain) Arx, Proc. K. Ned. Akad. Wet., Ser. C, Biol. Med. Sci. 76: 2931973.

EPreussia cylindrica Malloch \& Cain, Canadian Journal of Botany 50: 681972.

Figs 32-35

三Pychnidiophora cylindrica (Malloch \& Cain) Guarro, CBS Biodiv. Ser. 10: 3652012.
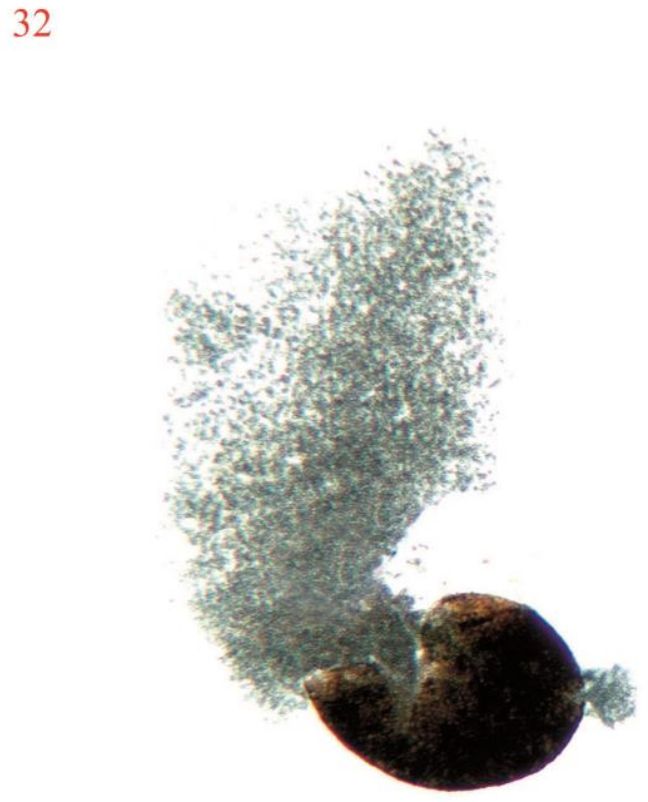

Fig 32 - Westerdykella cylindrica. Pseudothecium extruding ascospores - Bar $=100 \mu \mathrm{m}$.

Material examined - Lucca, Viareggio, 43d 52' 48.9462" 10d 13' 56.9166", 0 m a.s.1., dozens of scattered or gregarious, sometimes crowded, superficial or semi-immersed specimens on horse (Equus caballus) dung placed on sand of coastal dunes, 31 Mar 2013, G. Cacialli, CLSM 005.13 .

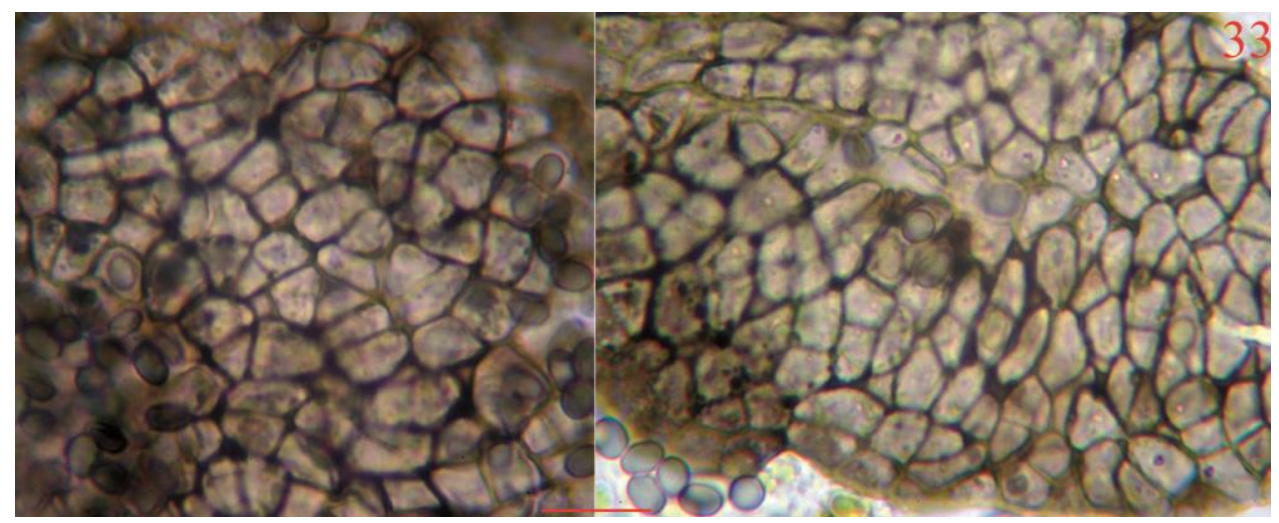

Fig 33 - Westerdykella cylindrica. Details of exoperidium - Bar $=15 \mu \mathrm{m}$.

Pseudothecia cleistothecioid, subglobose to pulvinate, sometimes slightly depressed, 100$150 \mu \mathrm{m}$ diam., asperulate, membranous, shining black by reflected light, dark brown by transmitted light. Peridium thin, two-layered, pseudoparenchymatous: exostratum a single layer of thick-walled, brown, polygonal cells, 5-9 $\times 4-7 \mu \mathrm{m}$; endoperidium with pale, slightly larger, polygonal cells. Pseudoparaphyses absent. Asci unitunicate, irregularly disposed, ephemeral, usually 32-spored from the very beginning, cylindric-clavate to clavate, $17-25 \times 9-10 \mu \mathrm{m}$, thick-walled at first, thinner at 


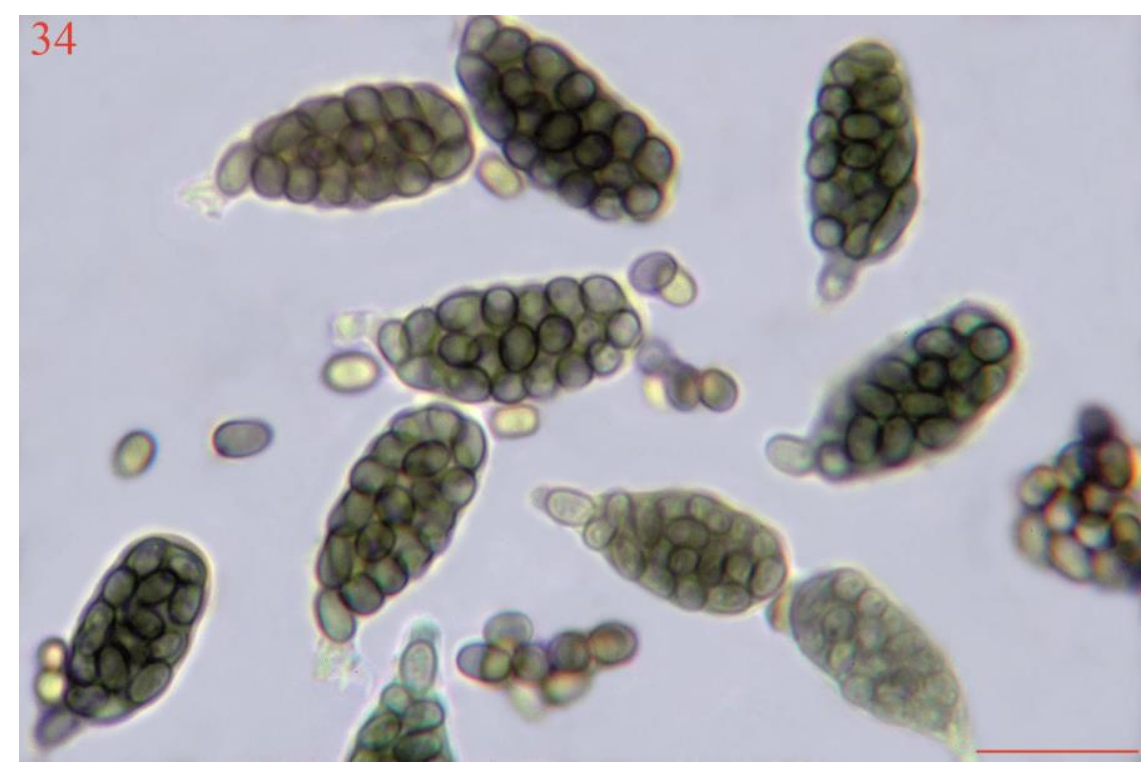

Fig 34 - Westerdykella cylindrica. 32-spores asci andi free ascospores - Bar $=15 \mu \mathrm{m}$.

maturity, lacking an apical apparatus, rounded at the apex, abruptly contracted at the base in a short, lobate stalk. Ascospores one-celled, (4-) 4.5-5 (-5.2) × 2.8-3.2 $\mu \mathrm{m}$, smooth, hyaline at first, pale grey at maturity, mostly short cylindrical with rounded ends (oblong), but also ellipsoidal or sometimes slightly asymmetrical and somewhat concave at one side (suballantoid), rarely narrowly ovoid or larger $(6.5-9 \times 3.5-5 \mu \mathrm{m})$, then dumb-bell shaped (with a bilateral middle constriction) or broadly ovoid, crowded inside the asci, thick-walled, without a germ pore or a germ slit and a gelatinous perisporium. Asexual state Phoma-like: pycnidia subglobose, more or less the same size as the cleistothecia, dark brown, with a circular ostiole about $20 \mu \mathrm{m}$ diam., smooth; pycnidial peridium pseudoparenchymatous, two-layered, with an exostratum of dark brown, thick-walled, polygonal cells, 4-10 $\times 4-8 \mu \mathrm{m}$, covered with numerous, septate, dark brown hyphae, 2-3 $\mu \mathrm{m}$ diam. Conidia 2.5-4 × 1.5-2 $\mu \mathrm{m}$, ellipsoidal to cylindric-ellipsoid or narrowly ovoid, sometimes slightly longitudinally asymmetrical, very pale yellow, smooth, with some droplets.

Notes - I have called Phoma-like, rather than Phoma Sacc., the asexual state of my W. cylindrica collection because phylogenetic studies (de Gruyter et al. 2013) proved Phoma to be polyphyletic and restricted to taxa in Didymellaceae Gruyter et al. The other Phoma spp. outside this family were recombined. Phoma capitulum and P. minutispora, nesting close to Westerdykella ornata Stolk in Sporormiaceae Munk, were respectively recombined in Westerdykella capitulum (V.H. Pawar et al.) Gruyter et al. and W. minutispora (P.N. Mathur ex Gruyter \& Noordel.) Gruyter et al.

The genus Westerdykella was erected by Stolk (1955) to accomodate a single species isolated from soil, W. ornata, with glabrous, dark cleistothecia, ephemeral, subglobose, 32-spored asci, brown, globose or subglobose ascospores ornamented with spiral bands and lacking both germ pores and slits, and no asexual state.

Another species isolated from soil, W. globosa (J.N. Rai \& J.P. Tewari) Tad. Ito \& Nakagiri, with 32-spored asci and globose ascospores with a single semicircular spiral band (Rai \& Tewari 1962, Ito \& Nakagiri 1995), was later accomodated in this genus.

Clum (1955) erected the genus Pycnidiophora and designated P. dispersa Clum as type species. The morphological features of this taxon fully match those of Westerdykella, except for having smooth, oblong-reniform ascospores and a pycnidial asexual state.

Other species were later erected in Pycnidiophora or recombined in this genus (Thomson \& Backus 1966, Mukerji \& Ranga Rao 1969, Guarro et al. 2012), all with 32-spored asci, but some without an imperfect state, some else with cylindrical or claviform asci and/or with cylindric to reniform ascospores. 


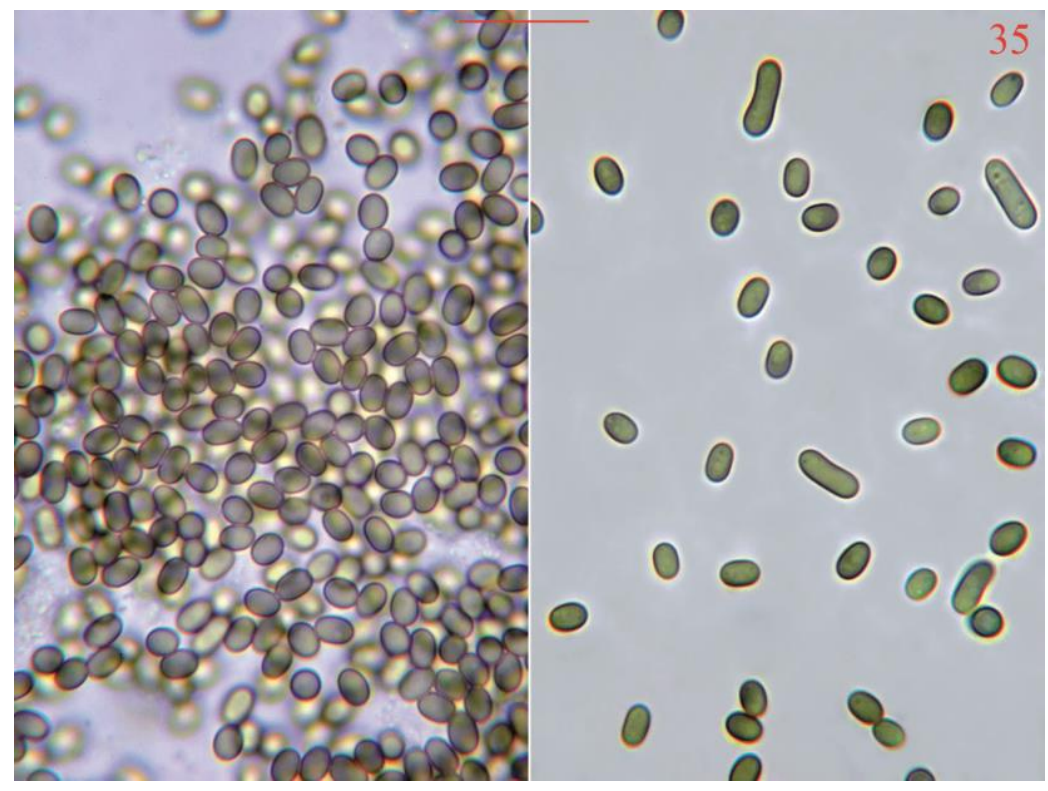

Fig 35 - Westerdykella cylindrica. Free ascospores - Bar $=15 \mu \mathrm{m}$.

Von Arx (1975) established the genus Eremodothis, recombining in this genus a single species, Thielavia angulata A.C. Das, with most morphological features very similar to Westerdykella and Pycnidiophora but with spherical, 8-spored asci and pyramidal to stellate ascospores with rounded ends (Das 1962, Guarro et al. 2012).

Recent phylogenetic studies (Kruys et al. 2006, Kruys \& Wedin 2009, Zhang et al. 2009, 2012, Ebead et al. 2012) proved that Pleosporales Luttr. ex M.E. Barr and Sporormiaceae (in Pleosporales) form well-supported monophyletic groups, Eremodothis belongs to Sporormiaceae, and Pycnidiophora and Eremodothis nest within Westerdykella and so should be treated as synonymous to the latter. Before then, others (Cejp \& Milko 1964, von Arx \& Storm 1967, von Arx 1981b) had suggested the synonymy between Pycnidiophora and Westerdykella, whereas von Arx \& van der Aa (1987) and Barr (2000) accepted Westerdykella only for species with globose, spirally banded ascospores. Kirk et al. (2008) and Guarro et al. (2012) still keep Emerodothis and Pycnidiophora independent from Westerdykella.

W. cylindrica is characterised by cylindric-clavate, 32-spored asci, smooth and mostly cylindrical ascospores, and a Phoma asexual state (Malloch \& Cain 1972, Ebead et al. 2012).

Based on ITS rDNA sequences (Ebead et al. 2012), W. cylindrica forms a sister clade with W. reniformis G.A. Ebead \& D.P., whereas in a multigene analysis of ITS, nLSU rDNA, and $\beta$ tubulin sequences it forms a well-supported clade with W. nigra (Routien) Arx. W. reniformis differs, however, in lacking a pycnidal asexual state and having globose or subglobose asci and reniform ascospores with a strong central groove (Ebead et al. 2012).

W. nigra shares cylindric-claviform asci with $W$. cylindrica, but in the former the ascospores are parallel to the ascus lenght and transversely three-septate in the early stages, soon separating in ellipsoid part-spores. Besides in W. nigra asci are larger $(30-40 \times 9-11 \mu \mathrm{m})$ and gently narrow towards their base, numerous ephemeral pseudoparaphyses are present, whereas an asexual state is absent (Routien 1956, Cain 1961).

A special mention deserves Pycnidiophora (= Westerdykella) cylindrispora (Lodha) Guarro, which has morphological features very similar to W. nigra, but differs in having slightly smaller asci $(23-31 \times 7-8 \mu \mathrm{m})$ with a longer stalk, and cylindric-oval rather than ellipsoid ascospore segments (Lodha 1971). According to Guarro et al. (2012) this species could be conspecific with W. nigra.

We refer to Guarro et al. (2012) for synonyms in Westerdykella and for the main differences between W. cylindrica and other Westerdykella spp., and to Ebead et al. (2012) for the most updated key to this genus (inclusive of Pycnidiophora and Emerodothis). 
Westerdykella spp. have mostly been isolated from soil (Routien 1956, Chattopadhyay \& Das Gupta 1957, Cain 1961, Mathur \& Thirumalachar 1962, Rai \& Tewari 1962, von Arx \& Storm 1967, Montemartini Corte 1968, Ahmad \& Sultana 1973, Lee \& Baker 1973, Mukerji \& Saxena 1975, Ito \& Nagakiri 1995), also from sediments of fresh (Milko 1965, Mishra 1995) or saline (ElSharouny et al. 2009) water, sometimes from vegetable matter (Clum 1955, Cain 1961, Ebead et al. 2012). As far as we know, only W. cylindrica and W. cylindrispora have been recorded, rarely and exclusively, from dung (Lodha 1971, Malloch \& Cain 1972, Bell 2005).

W. cylindrica, like most Westerdykella spp. was proved to produce compounds with antibiotic activity (Ebead et al. 2012).

\section{Acknowledgements}

The author thanks L. Levorato and all friends who provided him with part of the material subject of this work.

\section{References}

Abdullah SK, Alutby SD. 1999 - Additions to coprophilous fungi og Iraq V: cleistothecial ascomycetes. Journal of Basrah Researches 18, 81-86.

Ahmad S, Sultana K. 1973 - Contributions to the fungi of West Pakistan XVI. Biologia 19, 1-26.

Ahmed SI, Cain RF. 1972 - Revision of the genera Sporormia and Sporormiella. Canadian Journal of Botany 50, 419-477. http://dx.doi.org/10.1139/b72-061

Ahmed SI, Ismail ALS, Abdullah SK, 1971 - Contribution to the fungi of Iraq II. Coprophilous fungi. Bulletin of the Biological Research Centre 5, 16-32.

Ames LM. 1963 - A monograph of the Chaetomiaceae. United States Army Research and Development series 2.

Asad F, Ahmad SI. 1968 - Coprophilous fungi of west Pakistan. Part II. - Karachi. Pakistan Journal of Science and Industrial Research 11, 284-287.

Barr ME. 1990 - Prodromus to nonlichenized, pyrenomycetous members of class Hymenoascomycetes. Mycotaxon 39, 43-184.

Barr ME. 2000 - Notes on coprophilous bitunicate ascomycetes. Mycotaxon 76, 105-112.

Barrasa Gonzales JM. 1985 - Contribución al estudio taxonómico, ecológico y corológico de los Ascomycetes coprófilos en España. Universidad de Alcalá de Henares (degree thesis, 621 pp.).

Bell A. 2005 - An illustrated guide to the coprophilous Ascomycetes of Australia. CBS Biodiversity Series 3, 1-172.

Cacialli G, Caroti V, Doveri F. 1995 - Funghi fimicoli e rari o interessanti del litorale toscano. Schede di Micologia 1. A.M.B. Fondazione Centro Studi Micologici. Vicenza.

Cain RF. 1934 - Studies of coprophilous Sphaeriales in Ontario. University of Toronto Studies Mycological Series 38, 1-126.

Cain RF. 1957 - Studies of coprophilous Ascomycetes - VI. Species from the Hudson Bay area. Canadian Journal of Botany 35, 255-265.

Cain RF. 1961 - Studies of coprophilous Ascomycetes - VII. Preussia. Canadian Journal of Botany 39, 1633-1666.

Cannon PF, Kirk PM. 2007 - Fungal families of the world. CABI, U.K.

Cejp K, Milko AA. 1964 - Genera of the Eurotiaceae with 32 ascospores. I. Westerdykella. Česká Mykologie 18, 82-84.

Chattopadhyay SB, Das Gupta C. 1957 - Thielavia indica sp. nov. Transactions of the British Mycological Society 40, 277-278.

Clum FM. 1955 - A new genus in the Aspergillaceae. Mycologia 47, 899-901.

Cribb AB. 1994 - New records of fungi on dung from Cape York Peninsula, Queensland. Proceedings of the Royal Society of Queensland 104, 19-24.

Das AC. 1962 - New species of Thielavia and Sordaria. Transactions of the British Mycological 
Society $45,545-548$.

de Gruyter J, Woudenberg JHC, Aveskamp MM, Verkley GJM, Groenewald JZ, Crous PW. 2013 Redisposition of Phoma-like anamorphs in Pleosporales. Studies in Mycology 75, 1-36.

Delgado Avila AE, Piñero Chávez AJ, Urdaneta García LM. 2001a - Hongos coprofílicos del estado Zulia, Venezuela. Clases: Plectomycetes y Discomycetes. División Ascomycota. Revista Científica FCV-LUZ 11, 297-305.

Delgado Avila AE, Piñero Chávez AJ, Urdaneta García LM. 2001b - Two species representing a new genus of Microascaceae, from Venezuela. Revista Científica FCV-LUZ 11, 501-504.

Doveri F. 2004 - Fungi Fimicoli Italici. A.M.B. Fondazione Centro Studi Micologici. Vicenza.

Doveri F. 2011 - Addition to "Fungi Fimicoli Italici": An update on the occurrence of coprophilous Basidiomycetes and Ascomycetes in Italy with new records and descriptions. Mycosphere 2, $331-427$.

Doveri F. 2012 - Coprophilous discomycetes from the Tuscan archipelago (Italy). Description of two rare species and a new Trichobolus. Mycosphere 3, 503-522. Doi 10.5943 /mycosphere/3/4/13

Doveri F, Caroti V, Cacialli G. 1997 - An exceptional finding from horse dung: Enterocarpus grenotii. Contribution to the study of fimicolous fungi - XXVI. Bollettino del Gruppo Micologico G. Bresadola, Nuova serie 40 (2-3), 187- 190.

Ebead GA, Overy DP, Berrue F, Kerr RG. 2012 - Westerdykella reniformis sp. nov., producing the antibiotic metabolites melinacidin IV and chetracin B. IMA Fungus 3, 189-201.

El-Sharouny H, Gherbawy YAMH, Abdel-Aziz F. 2009 - Fungal diversity in brackish and saline lakes in Egypt. Nova Hedwigia 89, 437-450.

Ellis JB, Everhart BM. 1897 - New species of fungi from various localities. Bulletin of the Torrey Botanical Club 24, 125-137.

Fort F, Cano J, Guarro J, Gené J. 1990 - Contribución al estudio de los ascomicetos del suelo de España. XI. Boletín d la Sociedad Micológica de Madrid 14, 61-74.

Furuya K, Udagawa SI. 1972 - Coprophilous pyrenomycetes from Japan - II. Journal of General and Applied Microbiology 18, 455-467.

Furuya K, Udagawa SI 1973 - Coprophilous pyrenomycetes from Japan - III. Transactions of the Mycological Society of Japan 14, 7-30.

Gilgado F, Gené J, Cano J, Guarro J. 2007 - Reclassification of Graphium tectonae as Parascedosporium tectonae gen. nov., comb. nov., Pseudallescheria africana as Petriellopsis africana gen. nov., comb. nov. and Pseudallescheria fimeti as Lophotrichus fimeti comb. nov. International Journal of Systematic and Evolutionary Microbiology 57, 2171-2178.

Griffiths D. 1901 - The North American Sordariaceae. Memoires of the Torrey Botanical Club 11, $1-134$.

Guarro J. 1983 - A new coprophilous species of Rhytidospora. Mycologia 75, 927-930. http://dx.doi.org/10.2307/3792790

Guarro J, Gené J, Stchigel AM, Figueras MJ. 2012 - Atlas of soil Ascomycetes. CBS Biodiversity Series 10, Utrecht.

Hibbett DS et al. 2007 - A higher-level phylogenetic classification of the fungi. Mycological Research 111, 509-547. http://dx.doi.org/10.1016/j.mycres.2007.03.004

Issakainen J, Jalava J, Hyvönen J, Sahlberg N, Pirnes T, Campbell CK. 2003 - Relationships of Scopulariopsis based on LSU rDNA sequences. Medical Mycology 41, 31-42.

Issakainen J, Jalava J, Saari J, Campbell CK. 1999 - Relationship of Scedosporium prolificans with Petriella confirmed by partial LSU rDNA sequences. Mycological Research 103, 11791184. http://dx.doi.org/10.1017/S0953756299008333

Ito T, Nakagiri A. 1995 - Westerdykella globosa, a proposal for a new combination. Mycoscience 36, 361-363. http://dx.doi.org/10.1007/BF02268614

Jeng RS, Cain RF. 1977 - Rhytidospora, a new cleistocarpous genus of the Melanosporaceae. Mycotaxon 5, 278-282. 
Khan RS, Cain RF. 1979 - The genera Sporormiella et Sporormia in East Africa. Canadian Journal of Botany 57, 1174-1186. http://dx.doi.org/10.1139/b79-141

Kirk PM, Cannon PF, Minter DW, Stalpers JA. 2008 - Dictionary of the fungi, 10th edition. CABI Publishing, UK.

Krug JC, Jeng RS. 1979 - Rhytidospora and Pteridiosperma, gen. nov. (Melanosporaceae). Mycotaxon 10, 41-45.

Kruys $\AA$, Ericson L. 2008 - Species richness of coprophilous ascomycetes in relation to variable food intake by herbivores. Fungal Diversity 30, 73-81.

Kruys $\AA$, Eriksson OE, Wedin M. 2006 - Phylogenetic relationships of coprophilous Pleosporales (Dothideomycetes, Ascomycota), and the classification of some bitunicate taxa of unknown position. Mycological Research 110, 527-536. http://dx.doi.org/10.1016/j.mycres.2006.03.002

Kruys $\AA$, Wedin M. 2009 - Philogenetic relationships and an assessment of traditionally used taxonomic characters in the Sporormiaceae (Pleosporales, Dothideomycetes, Ascomycota), utilising multi-gene phylogenies. Systematics and Biodiversity 7, 465-478. http://dx.doi.org/10.1017/S1477200009990119

Lécuru C. 2013 - Les champignons fimicoles de la région Nord-Pas-de-Calais (France). Inventaire annoté. 1. Ascomycota et Zygomycota. Documents Mycologiques 35, 191-288.

Lee BKH, Baker GE. 1973 - Fungi associated with the roots of red mangrove, Rhizophora mangle. Mycologia 65, 894-906. http://dx.doi.org/10.2307/3758522

Locquin-Linard M. 1977 - A propos des genres non ostiolés placés dans la famille des Microascaceae (Ascomycètes). Revue de Mycologie (Paris) 41, 509-523.

Lodha BC. 1971 - Studies on coprophilous fungi IV. Some cleistothecial Ascomycetes. Journal of the Indian Botanical Society 50, 196-208.

Lumbsch HT, Huhndorf SM. 2007 - Outline of Ascomycota - 2007. Myconet 13, 1-58.

Lundqvist N. 1997 - Fungi fimicolae exsiccati. Thunbergia 25 (4-5), $\mathrm{n}^{\circ} 76-125$.

Malloch D. 1970 - New concepts in the Microascaceae illustrated by two new species. Mycologia 62, 727-740. http://dx.doi.org/10.2307/3757662

Malloch D, Cain RF. 1972 - New species and combinations of cleistothecial ascomycetes. Canadian Journal of Botany 50, 61-72. http://dx.doi.org/10.1139/b72-011

Massee G, Salmon ES. 1901 - Researches on coprophilous fungi. Annals of Botany 15 (58), 313357.

Mathur PN, Thirumalachar MJ. 1962 - Studies on some Indian soil Fungi-II. On some new or interesting Ascomycetes. Sydowia 16, 46-57.

Matsushima T. 1975 - Icones microfungorum a Matsushima lectorum. Published by the Author, Kobe, Japan.

McGinnis MR, Padhye AA, Ajello L. 1982 - Pseudallescheria Negroni et Fischer, 1943 and its later synonym Petriellidium Malloch, 1970. Mycotaxon 14, 94-102.

Milko AA. 1965 - Species nova et rarae eurotiacearum in aqua inventae. Novitates Systematicae Plantarum Non Vascularium, 122-125.

Minoura K. 1969 - Notes on some Ascomycetes of East Africa. Transactions of the Mycological Society of Japan 10, 41-46.

Mishra RC. 1995 - Distribution of microfungi in the transitional zone of a subtropical lake in India. Canadian Journal of Botany 73, 512-516. http://dx.doi.org/10.1139/b95-052

Montemartini Corte A. 1968 - Eurotiaceae trovate in risaia. Giornale Botanico Italiano 102, 55-65. http://dx.doi.org/10.1080/11263506809426690

Moyne G, Petit J. 2006-2007 - Quelques ascomycetes coprophiles recoltes en France et en particulier dans le departement du Doubs. Bulletin de la Société d'Histoire Naturelle du Doubs 91, 49-114.

Mukerji KG, Ranga Rao V. 1969 - Fungi of Delhi, XIII. Two interesting records from soil. Journal of General and Applied Microbiology 15, 261-265. http://dx.doi.org/10.2323/jgam.15.261

Mukerji KG, Saxena AS. 1975 - Notes on Achaetomium, Anixiella, Boothiella, Chaetomidium, 
Lophotrichus, Pseudeurotium, Pycnidiophora and the classification of the Chaetomiales. Nova Hedwigia Beih. 47, 373-404.

Munk A. 1957 - Danish Pyrenomycetes. Dansk Botanisk Arkiv 17, 1-491.

Niessl GV. 1878 - Die arten der Pyrenomycetengattung Sporormia de Not.. Österreichische Botanische Zeitschrift 28, 95-98. http://dx.doi.org/10.1007/BF01627128

Parker AD. 1979 - Associations between coprophilous ascomycetes and fecal substrates in Illinois. Mycologia 71, 1206-1214. http://dx.doi.org/10.2307/3759107

Pathak RK, Agrawal SC. 1974 - Two more additions to Indian ascomycetes. Current Science 43, 440.

Piontelli EL, Cruz RC, Alicia Toro Santa-maria M. 2006 - Coprophilous fungal community of wild rabbit in a park of a hospital (Chile): a taxonomic approach. Boletín Micológico 21, 1-17.

Rai JN, Tewari JP. 1962 - Pseudeurotium globosum, a new ascomycete from Indian soils. Mycopathologia et Mycologia Applicata 16, 90-96. http://dx.doi.org/10.1007/BF02136184

Rainer J, de Hoog GS. 2006 - Molecular taxonomy and ecology of Pseudallescheria, Petriella and Scedosporium prolificans (Microascaceae) containing opportunistic agents on humans. Mycological Research 110, 151-160. http://dx.doi.org/10.1016/j.mycres.2005.08.003

Ribeiro Melo RF, Bezerra JL, Queiroz de Cavalcanti MA. 2012 - Diversity of coprophilous ascomycetes from captive wild animals in Dois Irmãos State Park, Brazil. Nova Hedwigia 94, 153-162. http://dx.doi.org/10.1127/0029-5035/2012/0094-0153

Richardson MJ. 2001 - Diversity and occurrence of coprophilous fungi. Mycological Research 105, 387-402. http://dx.doi.org/10.1017/S0953756201003884

Richardson MJ. 2004 - Coprophilous fungi from Iceland. Acta Botanica Islandica 14, 77-102.

Richardson MJ. 2008 - Records of French coprophilous fungi. Cryptogamie, Mycologie 29, 157177.

Richardson MJ. 2011 - Additions to the coprophilous Mycota of Iceland. Acta Botanica Islandica $15,23-49$.

Richardson MJ, Watling R. 1997 - Keys to fungi on dung. British Mycological Society, Stourbridge.

Routien JB. 1956 - A new species of Muellerella and its development. Bulletin of the Torrey Botanical Club 83, 403-409. http://dx.doi.org/10.2307/2483092

Seth HK. 1971 - The genus Lophotrichus Benjamin. Nova Hedwigia 19, 591-599.

Simmons EG. 1952 - Culture studies in the genera Pleospora, Clathrospora, and Leptosphaeria. Mycologia 44, 330-365.

Spegazzini C. 1878 - Fungi coprophili Veneti. Michelia 1, 222-238.

Spegazzini C. 1879 - Nova addenda ad mycologiam Venetam. Michelia 1, 453-487.

Stolk AC. 1955 - Emericellopsis minima sp. nov. and Westerdykella ornata gen. nov., sp. nov. Transactions of the British Mycological Society 38, 419-424. http://dx.doi.org/10.1016/S0007-1536(55)80046-0

Thompson TW, Backus MP. 1966 - Further notes on Pycnidiophora dispersa and Pseudeurotium multisporum. Mycologia 58, 650-655. http://dx.doi.org/10.2307/3757049

Tóth S. 1967 - Data to the knowledge of the coprophilous microscopic fungi in Hungary III. Annales Historico-Naturales Musei Nationalis Hungaricxi Pars Botanica 59, 117-123.

Treigiené A. 2004 - Koprofiliniai pirenomicetai ir lokuloaskomicetai lietuvoje. Sporormiella ir Preussia gentys. Botanica Lithuanica suppl. 6, 77-88.

Valldosera M. 1991 - Contribución al estudio de los hongos coprófilos - Ascomycotina - en España. Universitat Autonoma de Barcelona (unpublished degree thesis).

Valldosera M, Guarro J. 1990 - Estudios sobre hongos coprófilos aislados en España. XV. El género Preussia (Sporormiella). Boletín de la Sociedad Micológica de Madrid 14, 81-94.

Valldosera M, Guarro J. 1994 - Two new coprophilous ascomycetes from South America. Nova Hedwigia 58, 447-451.

Valldosera M, Guarro J, Figueras MJ. 1987 - Estudio sobre hongos coprófilos aislados en España. V. Ascomycetes. Revista Ibérica de Micología 4, 33-36. 
Valldosera M, Guarro J, Figueras MJ. 1991 - Two interesting coprophilous fungi from Spain. Mycological Research 95, 243-246. http://dx.doi.org/10.1016/S0953-7562(09)81020-4

von Arx JA. 1975 - On Thielavia angulata and some recently described Thielavia species. Kavaka 3, 33-36.

von Arx JA. 1978 - Notes on Microascaceae with the description of two new species. Persoonia 10, 23-31.

von Arx JA. 1981a - On Monilia sitophila and some families of ascomycetes. Sydowia 34, 13-29.

von Arx JA. 1981b - The genera of fungi sporulating in pure culture. Third edition. Cramer. Vaduz.

von Arx JA, Figueras MJ, Guarro J. 1988 - Sordariaceous ascomycetes without ascospore ejaculation. Nova Hedwigia 94, 1-104.

von Arx JA, Storm PK. 1967 - Über einige aus dem erdboden isolierte, zu Sporormia, Preussia und Westerdykella gehörende Ascomyceten. Persoonia 4, 407-415.

von Arx JA, van der Aa HA. 1987 - Spororminula tenerifae gen. et sp. nov. Transactions of the British Mycological Society 89, 117-120. http://dx.doi.org/10.1016/S0007-1536(87)800682

Wang YZ. 1992 - New records of coprophilous pyrenomycetes from Taiwan (I). Transactions of the Mycological Society R.O.C. 7 (3-4), 18-22.

Webster J, Lucas MT. 1961 - Observations on British species of Pleospora. II. Transactions of the British Mycological Society 44, 417-436. http://dx.doi.org/10.1016/S0007-1536(61)800363

Wehmeyer LE. 1949 - Studies in the genus Pleospora. I. Mycologia 41, 565-593. http://dx.doi.org/10.2307/3755077

Wehmeyer LE. 1951 - Studies in the genus Pleospora. III. Mycologia 43, 34-53. http://dx.doi.org/10.2307/3755429

Welt P, Heine N. 2006 - Beiträge zur Kenntnis coprophiler Pilze (2) - Dungbewohnende Pilze Thüringens: Teil 1. Hoher Artenreichtum coprophiler Pilze in einem Schutzgebiet Indicator für eine intakte Natur ? Boletus 29, 81-92.

Wilson CM. 1947 - Coprophilous ascomycetes of Virginia. Mycologia 39, 374-377. http://dx.doi.org/10.2307/3755215

Zhang N, Castlebury LA, Miller AN, Huhndorf SM, Schoch CL, Seifert KA, Rossman AY, Rogers JD, Kohlmeyer J, Volkmann-Kohlmeyer B, Sung GH. 2006 - An overview of the systematics of the Sordariomycetes based on a four-gene phylogeny. Mycologia 98, 10761087. http://dx.doi.org/10.3852/mycologia.98.6.1076

Zhang Y, Crous PW, Schoch CL, Hyde KD. 2012 - Pleosporales. Fungal Diversity 53, 1-221. http://dx.doi.org/10.1007/s13225-011-0117-x

Zhang Y, Schoch CL, Fournier J, Crous PW, de Gruyter J, Woudenberg JH, Hirayama K, Tanaka K, Pointing SB, Spatafora JW, Hyde KD. 2009 - Multi-locus phylogeny of Pleosporales: a taxonomic, ecological and evolutionary re-evaluation. Studies in Mycology 64, 85-102. 